\title{
Vector-Valued Polynomials and a Matrix Weight Function with $B_{2}$-Action
}

Charles F. DUNKL

Department of Mathematics, University of Virginia, PO Box 400137, Charlottesville VA 22904-4137, USA

E-mail: cfd5z@virginia.edu

URL: http://people.virginia.edu/ cfd5z/home.html

Received October 16, 2012, in final form January 23, 2013; Published online January 30, 2013

http://dx.doi.org/10.3842/SIGMA.2013.007

\begin{abstract}
The structure of orthogonal polynomials on $\mathbb{R}^{2}$ with the weight function $\mid x_{1}^{2}-$ $\left.x_{2}^{2}\right|^{2 k_{0}}\left|x_{1} x_{2}\right|^{2 k_{1}} e^{-\left(x_{1}^{2}+x_{2}^{2}\right) / 2}$ is based on the Dunkl operators of type $B_{2}$. This refers to the full symmetry group of the square, generated by reflections in the lines $x_{1}=0$ and $x_{1}-x_{2}=0$. The weight function is integrable if $k_{0}, k_{1}, k_{0}+k_{1}>-\frac{1}{2}$. Dunkl operators can be defined for polynomials taking values in a module of the associated reflection group, that is, a vector space on which the group has an irreducible representation. The unique 2-dimensional representation of the group $B_{2}$ is used here. The specific operators for this group and an analysis of the inner products on the harmonic vector-valued polynomials are presented in this paper. An orthogonal basis for the harmonic polynomials is constructed, and is used to define an exponential-type kernel. In contrast to the ordinary scalar case the inner product structure is positive only when $\left(k_{0}, k_{1}\right)$ satisfy $-\frac{1}{2}<k_{0} \pm k_{1}<\frac{1}{2}$. For vector polynomials $\left(f_{i}\right)_{i=1}^{2},\left(g_{i}\right)_{i=1}^{2}$ the inner product has the form $\iint_{\mathbb{R}^{2}} f(x) K(x) g(x)^{T} e^{-\left(x_{1}^{2}+x_{2}^{2}\right) / 2} d x_{1} d x_{2}$ where the matrix function $K(x)$ has to satisfy various transformation and boundary conditions. The matrix $K$ is expressed in terms of hypergeometric functions.
\end{abstract}

Key words: matrix Gaussian weight function; harmonic polynomials

2010 Mathematics Subject Classification: 33C52; 42C05; 33C05

\section{Introduction}

The algebra of operators on polynomials generated by multiplication and the Dunkl operators associated with some reflection group is called the rational Cherednik algebra. It is parametrized by a multiplicity function which is defined on the set of roots of the group and is invariant under the group action. For scalar-valued polynomials there exists a Gaussian-type weight function which demonstrates the positivity of a certain bilinear form on polynomials, for positive values (and a small interval of negative values) of the multiplicity function. The algebra can also be represented on polynomials with values in an irreducible module of the group. In this case the problem of finding a Gaussian-type weight function and the multiplicity-function values for which it is positive and integrable becomes much more complicated. Here we initiate the study of this problem on the smallest two-parameter, two-dimensional example, namely, the group of type $B_{2}$ (the full symmetry group of the square).

Griffeth [7] defined and studied analogues of nonsymmetric Jack polynomials for arbitrary irreducible representations of the complex reflection groups in the family $G(r, 1, n)$. This paper introduced many useful methods for dealing with vector-valued polynomials. In the present paper we consider $B_{2}$, which is the member $G(2,1,2)$ of the family, but we use harmonic polynomials, rather than Griffeth's Jack polynomials because the former play a crucial part in the analysis of the Gaussian weight. There is a detailed study of the unitary representations of the rational Cherednik algebra for the symmetric and dihedral groups in Etingof and Stoica [6]. 
We begin with a brief discussion of vector-valued polynomials and the results which hold for any real reflection group. This includes the definition of the Dunkl operators and the basic bilinear form. The next section specializes to the group $B_{2}$ and contains the construction of an orthogonal basis of harmonic homogeneous polynomials, also a brief discussion of the radical. Section 4 uses this explicit basis to construct the appropriate analogue of the exponential function. Section 5 contains the derivation of the Gaussian-type weight function; it is a $2 \times 2$ matrix function whose entries involve hypergeometric functions. This is much more complicated than the scalar case. The method of solution is to set up a system of differential equations, find a fundamental solution and then impose several geometric conditions, involving behavior on the mirrors (the walls of the fundamental region of the group) to construct the desired solution.

\section{General results}

Suppose $R$ is a root system in $\mathbb{R}^{N}$ and $W=W(R)$ is the finite Coxeter group generated by $\left\{\sigma_{v}: v \in R_{+}\right\} \quad$ (where $\langle x, y\rangle:=\sum_{i=1}^{N} x_{i} y_{i},|x|:=\langle x, x\rangle^{1 / 2}, x \sigma_{v}:=x-2 \frac{\langle x, v\rangle}{\langle v, v\rangle} v$ for $x, y, v \in \mathbb{R}^{N}$ and $v \neq 0)$. Let $\kappa$ be a multiplicity function on $R(u=v w$ for some $w \in W$ and $u, v \in R$ implies $\kappa(u)=\kappa(v)$ ). Suppose $\tau$ is an irreducible representation of $W$ on a (real) vector space $V$ of polynomials in $t \in \mathbb{R}^{N}$ of dimension $n_{\tau}$. (There is a general result for these groups that real representations suffice, see $\left[1\right.$, Chapter 11].) Let $\mathcal{P}_{V}$ be the space of polynomial functions $\mathbb{R}^{N} \rightarrow V$, that is, the generic $f \in \mathcal{P}_{V}$ can be expressed as $f(x, t)$ where $f$ is a polynomial in $x, t$ and $f(x, t) \in V$ for each fixed $x \in \mathbb{R}^{N}$. There is an action of $W$ on $\mathcal{P}_{V}$ given by

$$
w f(x, t):=f(x w, t w), \quad w \in W .
$$

Define Dunkl operators on $\mathcal{P}_{V}$, for $1 \leq i \leq N$ by

$$
\mathcal{D}_{i} f(x, t):=\frac{\partial}{\partial x_{i}} f(x, t)+\sum_{v \in R_{+}} \kappa(v) \frac{f\left(x, t \sigma_{v}\right)-f\left(x \sigma_{v}, t \sigma_{v}\right)}{\langle x, v\rangle} v_{i} .
$$

There is an equivariance relation, for $u \in \mathbb{R}^{N}, w \in W$

$$
\sum_{i=1}^{N} u_{i} \mathcal{D}_{i} w=w \sum_{i=1}^{N}(u w)_{i} \mathcal{D}_{i} .
$$

Ordinary (scalar) polynomials act by multiplication on $\mathcal{P}_{V}$. For $1 \leq i, j \leq N$ and $f \in \mathcal{P}_{V}$ the basic commutation rule is

$$
\mathcal{D}_{i} x_{j} f(x, t)-x_{j} \mathcal{D}_{i} f(x, t)=\delta_{i j} f(x, t)+2 \sum_{v \in R_{+}} \kappa(v) \frac{v_{i} v_{j}}{|v|^{2}} f\left(x \sigma_{v}, t \sigma_{v}\right) .
$$

The abstract algebra generated by $\left\{x_{i}, \mathcal{D}_{i}: 1 \leq i \leq N\right\} \cup \mathbb{R} W$ with the commutation relations $x_{i} x_{j}=x_{j} x_{i}, \mathcal{D}_{i} \mathcal{D}_{j}=\mathcal{D}_{j} \mathcal{D}_{i},(2)$ and equivariance relations like (1) is called the rational Cherednik algebra of $W$ parametrized by $\kappa$; henceforth denoted by $\mathcal{A}_{\kappa}$. Then $\mathcal{P}_{V}$ is called the standard module of $\mathcal{A}_{\kappa}$ determined by the $W$-module $V$.

We introduce symmetric bilinear $W$-invariant forms on $\mathcal{P}_{V}$. There is a $W$-invariant form $\langle\cdot, \cdot\rangle_{\tau}$ on $V$; it is unique up to multiplication by a constant because $\tau$ is irreducible. The form is extended to $\mathcal{P}_{V}$ subject to $\left\langle x_{i} f(x, t), g(x, t)\right\rangle_{\tau}=\left\langle f(x, t), \mathcal{D}_{i} g(x, t)\right\rangle_{\tau}$ for $f, g \in \mathcal{P}_{V}$ and $1 \leq i \leq N$. To be more specific: let $\left\{\xi_{i}(t): 1 \leq i \leq n_{\tau}\right\}$ be a basis for $V$. Any $f \in \mathcal{P}_{V}$ has a unique expression $f(x, t)=\sum_{i} f_{i}(x) \xi_{i}(t)$ where each $f_{i}(x)$ is a polynomial, and then

$$
\langle f, g\rangle_{\tau}:=\sum_{i}\left\langle\xi_{i}(t),\left.\left(f_{i}\left(\mathcal{D}_{1}, \ldots, \mathcal{D}_{N}\right) g(x, t)\right)\right|_{x=0}\right\rangle_{\tau}, \quad g \in \mathcal{P}_{V} .
$$

The form satisfies $\langle f, g\rangle_{\tau}=\langle w f, w g\rangle_{\tau}=\langle g, f\rangle_{\tau}, w \in W$. 
This is a general result for standard modules of the rational Cherednik algebra, see [4]. The proof is based on induction on the degree and the eigenfunction decomposition of the operator $\sum_{i=1}^{N} x_{i} \mathcal{D}_{i}$. Indeed

$$
\sum_{i=1}^{N} x_{i} \mathcal{D}_{i} f(x, t)=\langle x, \nabla\rangle f(x, t)+\sum_{v \in R_{+}} \kappa(v)\left(f\left(x, t \sigma_{v}\right)-f\left(x \sigma_{v}, t \sigma_{v}\right)\right),
$$

where $\nabla$ denotes the gradient (so that $\langle x, \nabla\rangle=\sum_{i=1}^{N} x_{i} \frac{\partial}{\partial x_{i}}$ ). Because $\tau$ is irreducible there are integers $c_{\tau}(v)$, constant on conjugacy classes of reflections (namely, the values of the character of $\tau$ ) such that

$$
\sum_{v \in R_{+}} \kappa(v) f\left(x, t \sigma_{v}\right)=\gamma(\kappa ; \tau) f(x, t), \quad \gamma(\kappa ; \tau):=\sum_{v \in R_{+}} c_{\tau}(v) \kappa(v),
$$

for each $f \in \mathcal{P}_{V}$. The Laplacian is

$$
\begin{aligned}
\Delta_{\kappa} f(x, t) & :=\sum_{i=1}^{N} \mathcal{D}_{i}^{2} f(x, t) \\
& =\Delta f(x, t)+\sum_{v \in R_{+}} \kappa(v)\left\{2 \frac{\left\langle v, \nabla f\left(x, t \sigma_{v}\right)\right\rangle}{\langle x, v\rangle}-|v|^{2} \frac{f\left(x, t \sigma_{v}\right)-f\left(x \sigma_{v}, t \sigma_{v}\right)}{\langle x, v\rangle^{2}}\right\},
\end{aligned}
$$

where $\Delta$ and $\nabla$ denote the ordinary Laplacian and gradient, respectively. Motivated by the Gaussian inner product for scalar polynomials (case $\tau=1$ ) which is defined by

$$
\langle f, g\rangle_{G}:=c_{\kappa} \int_{\mathbb{R}^{N}} f(x) g(x) \prod_{v \in R_{+}}|\langle x, v\rangle|^{2 \kappa(v)} e^{-|x|^{2} / 2} d x,
$$

where $c_{\kappa}$ is a normalizing (Macdonald-Mehta) constant, and satisfies

$$
\langle f, g\rangle_{\tau}=\left\langle e^{-\Delta_{\kappa} / 2} f, e^{-\Delta_{\kappa} / 2} g\right\rangle_{G},
$$

we define a bilinear Gaussian form on $\mathcal{P}_{V}$ by

$$
\langle f, g\rangle_{G}:=\left\langle e^{\Delta_{\kappa} / 2} f, e^{\Delta_{\kappa} / 2} g\right\rangle_{\tau}
$$

Note $e^{-\Delta_{\kappa} / 2}:=\sum_{n=0}^{\infty} \frac{(-1 / 2)^{n}}{n !} \Delta_{\kappa}^{n}$ is defined for all polynomials since $\Delta_{\kappa}$ is nilpotent. From the relations

$$
\Delta_{\kappa}\left(x_{i} f(x, t)\right)=x_{i} \Delta_{\kappa} f(x, t)+2 \mathcal{D}_{i} f(x, t), \quad e^{-\Delta_{\kappa} / 2}\left(x_{i} f(x, t)\right)=\left(x_{i}-\mathcal{D}_{i}\right) e^{-\Delta_{\kappa} / 2} f(x, t),
$$

we find that $\left\langle\left(x_{i}-\mathcal{D}_{i}\right) f, g\right\rangle_{G}=\left\langle f, \mathcal{D}_{i} g\right\rangle_{G}$, for $1 \leq i \leq N$ and $f, g \in \mathcal{P}_{V}$. Thus the multiplier operator $x_{i}$ is self-adjoint for this form (since $x_{i}=\mathcal{D}_{i}+\mathcal{D}_{i}^{*}$ ). This suggests that the form may have an expression as an actual integral over $\mathbb{R}^{N}$, at least for some restricted set of the parameter values $\kappa(v)$. As in the scalar case harmonic polynomials are involved in the analysis of the Gaussian form. The equation

$$
\sum_{i=1}^{N}\left(x_{i} \mathcal{D}_{i}+\mathcal{D}_{i} x_{i}\right)=N+2\langle x, \nabla\rangle+2 \gamma(\kappa ; \tau)
$$


shows that

$$
\Delta_{\kappa}|x|^{2 m} f=2 m|x|^{2(m-1)}(2 m-2+N+2 \gamma(\kappa ; \tau)+2\langle x, \nabla\rangle) f+|x|^{2 m} \Delta_{\kappa} f,
$$

for $f \in \mathcal{P}_{V}$. For $n=0,1,2, \ldots$ let $\mathcal{P}_{V, n}=\left\{f \in \mathcal{P}_{V}: f(r x, t)=r^{n} f(x, t), \forall r \in \mathbb{R}\right\}$, the polynomials homogeneous of degree $n$, and let $\mathcal{H}_{V, \kappa, n}:=\left\{f \in \mathcal{P}_{V, n}: \Delta_{\kappa} f=0\right\}$, the harmonic homogeneous polynomials. As a consequence of the previous formula, for $m=1,2,3, \ldots$ and $f \in \mathcal{H}_{V, \kappa, n}$ one obtains

$$
\Delta_{\kappa}^{k}\left(|x|^{2 m} f(x, t)\right)=4^{k}(-m)_{k}(1-m-N / 2-\gamma(\kappa ; \tau)-n)_{k}|x|^{2 m-2 k} f(x, t) .
$$

(The Pochhammer symbol $(a)_{k}$ is defined by $(a)_{0}=1,(a)_{k+1}=(a)_{k}(a+k)$ or $(a)_{k}:=\prod_{i=1}^{k}(a+i-1)$. In particular $\left(\begin{array}{l}n \\ k\end{array}\right)=(-1)^{k} \frac{(-n)_{k}}{k !}$ and $(-n)_{k}=0$ for $n=0, \ldots, k-1$.) Thus $\Delta_{\kappa}^{k}\left(|x|^{2 m} f(x, t)\right)=0$ for $k>m$. With the same proofs as for the scalar case [5, Theorem 5.1.15] one can show (provided $\gamma(\kappa ; \tau)+\frac{N}{2} \neq 0,-1,-2, \ldots$ ): if $f \in \mathcal{P}_{V, n}$ then

$$
\begin{aligned}
& \pi_{\kappa, n} f:=\sum_{j=0}^{\lfloor n / 2\rfloor} \frac{1}{4^{j} j !(-\gamma(\kappa ; \tau)-n+2-N / 2)_{j}}|x|^{2 j} \Delta_{\kappa}^{j} f \in \mathcal{H}_{V, \kappa, n}, \\
& f=\sum_{j=0}^{\lfloor n / 2\rfloor} \frac{1}{4^{j} j !(\gamma(\kappa ; \tau)+N / 2+n-2 j)_{j}}|x|^{2 j} \pi_{\kappa, n-2 j}\left(\Delta_{\kappa}^{j} f\right) .
\end{aligned}
$$

From the definition of $\langle\cdot, \cdot\rangle_{\tau}$ it follows that $f \in \mathcal{P}_{V, m}, g \in \mathcal{P}_{V, n}$ and $m \neq n$ implies $\langle f, g\rangle_{\tau}=0$. Also if $f \in \mathcal{H}_{V, \kappa, m}, g \in \mathcal{H}_{V, \kappa, n}, m \neq n$ then $\left\langle|x|^{2 a} f,|x|^{2 b} g\right\rangle_{\tau}=0$ for any $a, b=0,1,2, \ldots$ This follows from the previous statement when $2 a+m \neq 2 b+n$, otherwise $n=m+2 a-2 b$ and assume $m<n$ (by symmetry of the form), thus $\left\langle|x|^{2 a} f,|x|^{2 b} g\right\rangle_{\tau}=\left\langle f, \Delta_{\kappa}^{a}|x|^{2 b} g\right\rangle_{\tau}=0$ because $a>b$. This shows that for generic $\kappa$ there is an orthogonal decomposition of $\mathcal{P}_{V}$ as a sum of $|x|^{2 m} \mathcal{H}_{V, \kappa, n}$ over $m, n=0,1,2, \ldots$ If $f, g \in \mathcal{H}_{V, \kappa, n}$ then

$$
\left\langle|x|^{2 m} f,|x|^{2 m} g\right\rangle_{\tau}=\left\langle f, \Delta_{\kappa}^{m}|x|^{2 m} g\right\rangle_{\tau}=4^{m} m !\left(\frac{N}{2}+\gamma(\kappa ; \tau)+n\right)_{m}\langle f, g\rangle_{\tau},
$$

so to find an orthogonal basis for $\mathcal{P}_{V, m}$ it suffices to find an orthogonal basis for each $\mathcal{H}_{V, \kappa, n}$.

The decomposition formula (5) implies the dimensionality result for $\mathcal{H}_{V, \kappa, n}$ (when $\gamma(\kappa ; \tau)+$ $\frac{N}{2} \notin-\mathbb{N}_{0}$ ). It is clear that $\operatorname{dim} \mathcal{P}_{V, n}=\operatorname{dim} \mathcal{P}_{\mathbb{R}, n} \operatorname{dim} V=\left(\begin{array}{c}N+n-1 \\ N-1\end{array}\right) \operatorname{dim} V$, and by induction (for $n \geq 1$ )

$$
\operatorname{dim} \mathcal{H}_{V, \kappa, n}=\operatorname{dim} \mathcal{P}_{V, n}-\operatorname{dim} \mathcal{P}_{V, n-2}=\left(\begin{array}{c}
N+n-2 \\
N-2
\end{array}\right) \frac{N+2 n-2}{N+n-2} \operatorname{dim} V .
$$

We will need a lemma about integrating closed 1 -forms. Consider an $N$-tuple $\mathbf{f}=\left(f_{1}, \ldots, f_{N}\right)$ $\in \mathcal{P}_{V}^{N}$ as a vector on which $W$ can act on the right. Say $\mathbf{f}$ is a closed 1 -form if $\mathcal{D}_{i} f_{j}-\mathcal{D}_{j} f_{i}=0$ for all $i, j$.

Lemma 1. Suppose $\mathbf{f}$ is a closed 1 -form and $1 \leq j \leq N$ then

$$
\mathcal{D}_{j} \sum_{i=1}^{N} x_{i} f_{i}(x, t)=(\langle x, \nabla\rangle+1+\gamma(\kappa ; \tau)) f_{j}(x, t)-\sum_{v \in R_{+}} \kappa(v)\left(\mathbf{f}\left(x \sigma_{v}, t \sigma_{v}\right) \sigma_{v}\right)_{j} .
$$


Proof. By the commutation relations (2)

$$
\begin{aligned}
\mathcal{D}_{j} \sum_{i=1}^{N} x_{i} f_{i}(x, t) & =\sum_{i=1}^{N}\left(x_{i} \mathcal{D}_{j} f_{i}+\delta_{i j} f_{i}+2 \sum_{v \in R_{+}} \kappa(v) \frac{v_{i} v_{j}}{|v|^{2}} f_{i}\left(x \sigma_{v}, t \sigma_{v}\right)\right) \\
& =\sum_{i=1}^{N} x_{i} \mathcal{D}_{i} f_{j}+f_{j}+2 \sum_{v \in R_{+}} \kappa(v) \frac{v_{j}}{|v|^{2}} \sum_{i=1}^{N} f_{i}\left(x \sigma_{v}, t \sigma_{v}\right) v_{i} .
\end{aligned}
$$

The calculation is finished with the use of (3).

Corollary 1. Suppose $\mathbf{f}$ is a closed 1-form, homogeneous of degree $n$ and

$$
\sum_{v \in R_{+}} \kappa(v) \mathbf{f}\left(x \sigma_{v}, t \sigma_{v}\right) \sigma_{v}=\lambda_{\kappa} \mathbf{f}(x, t)
$$

for some constant $\lambda_{\kappa}$ then

$$
\mathcal{D}_{j} \sum_{i=1}^{N} x_{i} f_{i}(x, t)=\left(n+1+\gamma(\kappa ; \tau)-\lambda_{\kappa}\right) f_{j}(x, t)
$$

for $1 \leq j \leq N$.

\section{The group $B_{2}$}

The rest of the paper concerns this group. The group $W\left(B_{2}\right)$ is the reflection group with positive root system $R_{+}=\{(1,-1),(1,1),(1,0),(0,1)\}$. The corresponding reflections are

$$
\sigma_{12}^{+}:=\left[\begin{array}{cc}
0 & 1 \\
1 & 0
\end{array}\right], \quad \sigma_{12}^{-}:=\left[\begin{array}{cc}
0 & -1 \\
-1 & 0
\end{array}\right], \quad \sigma_{1}:=\left[\begin{array}{cc}
-1 & 0 \\
0 & 1
\end{array}\right], \quad \sigma_{2}:=\left[\begin{array}{cc}
1 & 0 \\
0 & -1
\end{array}\right] .
$$

The values of $\kappa$ on the conjugacy classes $\left\{\sigma_{12}^{+}, \sigma_{12}^{-}\right\}$and $\left\{\sigma_{1}, \sigma_{2}\right\}$ will be denoted by $k_{0}$ and $k_{1}$, respectively. We consider the unique 2-dimensional representation $\tau$ and set $V:=\operatorname{span}\left\{t_{1}, t_{2}\right\}$. The generic element of $\mathcal{P}_{V}$ is $f(x, t)=f_{1}(x) t_{1}+f_{2}(x) t_{2}$. The reflections act on this polynomial as follows

$$
\begin{aligned}
& \sigma_{12}^{+} f(x, t)=f_{2}\left(x_{2}, x_{1}\right) t_{1}+f_{1}\left(x_{2}, x_{1}\right) t_{2}, \\
& \sigma_{12}^{-} f(x, t)=-f_{2}\left(-x_{2},-x_{1}\right) t_{1}-f_{1}\left(-x_{2},-x_{1}\right) t_{2}, \\
& \sigma_{1} f(x, t)=-f_{1}\left(-x_{1}, x_{2}\right) t_{1}+f_{2}\left(-x_{1}, x_{2}\right) t_{2}, \\
& \sigma_{2} f(x, t)=f_{1}\left(x_{1},-x_{2}\right) t_{1}-f_{2}\left(x_{1},-x_{2}\right) t_{2} .
\end{aligned}
$$

Here is the formula for $\mathcal{D}_{1}\left(\mathcal{D}_{2}\right.$ is similar $)$

$$
\begin{aligned}
\mathcal{D}_{1} f(x, t)= & \frac{\partial}{\partial x_{1}} f(x, t)+k_{1} \frac{f\left(x_{1}, x_{2},-t_{1}, t_{2}\right)-f\left(-x_{1}, x_{2},-t_{1}, t_{2}\right)}{x_{1}} \\
& +k_{0} \frac{f\left(x_{1}, x_{2}, t_{2}, t_{1}\right)-f\left(x_{2}, x_{1}, t_{2}, t_{1}\right)}{x_{1}-x_{2}} \\
& +k_{0} \frac{f\left(x_{1}, x_{2},-t_{2},-t_{1}\right)-f\left(-x_{2},-x_{1},-t_{2},-t_{1}\right)}{x_{1}+x_{2}} .
\end{aligned}
$$

Since the matrices for the reflections all have trace zero we find that $\gamma(\kappa ; \tau)=0$. We investigate the properties of $\langle\cdot, \cdot\rangle_{\tau}$ by constructing bases for each $\mathcal{H}_{V, \kappa, n}$; note $\operatorname{dim} \mathcal{H}_{V, \kappa, n}=4$ for $n \geq 1$. 
The form on $V$ is given by $\left\langle t_{i}, t_{j}\right\rangle_{\tau}=\delta_{i j}$. A convenient orthogonal decomposition is based on types defined by the action of $\left(\sigma_{1}, \sigma_{2}\right)$ : suppose $f$ satisfies $\sigma_{1} f=\varepsilon_{1} f, \sigma_{2} f=\varepsilon_{2} f$, then $f$ is of type $\mathrm{EE}, \mathrm{EO}, \mathrm{OE}$, OO if $\left(\varepsilon_{1}, \varepsilon_{2}\right)=(1,1),(1,-1),(-1,1),(-1,-1)$ respectively ( $\mathrm{E}$ is for even, $\mathrm{O}$ is for odd). Because the reflections are self-adjoint for the form $\langle\cdot, \cdot\rangle_{\tau}$ it follows immediately that polynomials of different types are mutually orthogonal. By formula (7) $\operatorname{dim} \mathcal{H}_{V, \kappa, n}=4$ for $n \geq 1$. The construction of the harmonic basis $\left\{p_{n, i}: n \geq 0,1 \leq i \leq 4\right\}$ is by a recurrence; the process starts at degree 1 . Set

$$
\begin{array}{ll}
p_{1,1}:=x_{1} t_{1}+x_{2} t_{2}, & p_{1,2}:=-x_{2} t_{1}+x_{1} t_{2}, \\
p_{1,3}:=x_{1} t_{1}-x_{2} t_{2}, & p_{1,4}:=-x_{2} t_{1}-x_{1} t_{2} .
\end{array}
$$

Thus $p_{1,1}, p_{1,3}$ are of type EE and $p_{1,2}, p_{1,4}$ are of type OO. Also $\left\langle p_{1,1}, p_{1,3}\right\rangle_{\tau}=0=\left\langle p_{1,2}, p_{1,4}\right\rangle_{\tau}$ because $\sigma_{12}^{+} p_{1,1}=p_{1,1}, \sigma_{12}^{+} p_{1,3}=-p_{1,3}, \sigma_{12}^{+} p_{1,2}=-p_{1,2}, \sigma_{12}^{+} p_{1,4}=p_{1,4}$. The same decompositions work in $\mathcal{H}_{V, \kappa, n}$ for each odd $n$. By direct computation we obtain

$$
\begin{aligned}
\left\langle p_{1,1}, p_{1,1}\right\rangle_{\tau} & =2\left(1-2 k_{0}-2 k_{1}\right), & \left\langle p_{1,2}, p_{1,2}\right\rangle_{\tau} & =2\left(1+2 k_{0}+2 k_{1}\right), \\
\left\langle p_{1,3}, p_{1,3}\right\rangle_{\tau} & =2\left(1+2 k_{0}-2 k_{1}\right), & \left\langle p_{1,4}, p_{1,4}\right\rangle_{\tau} & =2\left(1-2 k_{0}+2 k_{1}\right) .
\end{aligned}
$$

These values show that a necessary condition for the existence of a positive-definite inner product on $\mathcal{P}_{V}$ is $-\frac{1}{2}< \pm k_{0} \pm k_{1}<\frac{1}{2}$. Note this is significantly different from the analogous condition for scalar polynomials (that is, $\tau=1$ ), namely $k_{0}, k_{1}, k_{0}+k_{1}>-\frac{1}{2}$. The formulae become more readable by the use of

$$
k_{+}:=k_{0}+k_{1}, \quad k_{-}:=k_{1}-k_{0} .
$$

The recurrence starts at degree 0 by setting $p_{0,1}=p_{0,3}=t_{1}$ and $p_{0,2}=-p_{0,4}=t_{2}$ (in the exceptional case $n=0$ there are only two linearly independent polynomials).

Definition 1. The polynomials $p_{n, i} \in \mathcal{P}_{V, n}$ for $n \geq 1$ and $1 \leq i \leq 4$ are given by

$$
\left[\begin{array}{ll}
p_{2 m+1,1} & p_{2 m+1,3} \\
p_{2 m+1,2} & p_{2 m+1,4}
\end{array}\right]:=\left[\begin{array}{cc}
x_{1} & x_{2} \\
-x_{2} & x_{1}
\end{array}\right]\left[\begin{array}{ll}
p_{2 m, 1} & p_{2 m, 3} \\
p_{2 m, 2} & p_{2 m, 4}
\end{array}\right], \quad m \geq 0
$$

and

$$
\begin{aligned}
& {\left[\begin{array}{l}
p_{2 m, 1} \\
p_{2 m, 2}
\end{array}\right]:=\frac{1}{2 m-1}\left[\begin{array}{cc}
x_{1} & x_{2} \\
-x_{2} & x_{1}
\end{array}\right]\left[\begin{array}{l}
\left(2 m-1+2 k_{-}\right) p_{2 m-1,3} \\
\left(2 m-1-2 k_{-}\right) p_{2 m-1,4}
\end{array}\right],} \\
& {\left[\begin{array}{l}
p_{2 m, 3} \\
p_{2 m, 4}
\end{array}\right]:=\frac{1}{2 m-1}\left[\begin{array}{cc}
x_{1} & x_{2} \\
-x_{2} & x_{1}
\end{array}\right]\left[\begin{array}{l}
\left(2 m-1+2 k_{+}\right) p_{2 m-1,1} \\
\left(2 m-1-2 k_{+}\right) p_{2 m-1,2}
\end{array}\right],}
\end{aligned}
$$

for $m \geq 1$.

The construction consists of two disjoint sequences

$$
\begin{aligned}
& {\left[\begin{array}{l}
p_{1,1} \\
p_{1,2}
\end{array}\right] \stackrel{1 \pm 2 k_{+}}{\rightarrow}\left[\begin{array}{l}
p_{2,3} \\
p_{2,4}
\end{array}\right] \rightarrow\left[\begin{array}{l}
p_{3,3} \\
p_{3,4}
\end{array}\right] \stackrel{3 \pm 2 k_{-}}{\rightarrow}\left[\begin{array}{l}
p_{4,1} \\
p_{4,2}
\end{array}\right] \rightarrow\left[\begin{array}{l}
p_{5,1} \\
p_{5,2}
\end{array}\right] \stackrel{5 \pm 2 k_{+}}{\rightarrow},} \\
& {\left[\begin{array}{l}
p_{1,3} \\
p_{1,4}
\end{array}\right] \stackrel{1 \pm 2 k_{-}}{\rightarrow}\left[\begin{array}{l}
p_{2,1} \\
p_{2,2}
\end{array}\right] \rightarrow\left[\begin{array}{l}
p_{3,1} \\
p_{3,2}
\end{array}\right] \stackrel{3 \pm 2 k_{+}}{\rightarrow}\left[\begin{array}{l}
p_{4,3} \\
p_{4,4}
\end{array}\right] \rightarrow\left[\begin{array}{l}
p_{5,3} \\
p_{5,4}
\end{array}\right] \stackrel{5 \pm 2 k_{-}}{\rightarrow} .}
\end{aligned}
$$

The typical steps are $\left[p_{2 m+1, *}\right]=X\left[p_{2 m, *}\right]$ and $\left[p_{2 m+2, *}\right]=X C_{2 m+1}\left[p_{2 m+1, o}\right](*$ and $\circ$ denote a 2-vector with labels 1,2 or 3,4 as in the diagram) where

$$
X=\left[\begin{array}{cc}
x_{1} & x_{2} \\
-x_{2} & x_{1}
\end{array}\right], \quad C_{2 m+1}=\left[\begin{array}{cc}
\frac{2 m+1+2 \lambda}{2 m+1} & 0 \\
0 & \frac{2 m+1-2 \lambda}{2 m+1}
\end{array}\right],
$$

where $\lambda=k_{+}$or $k_{-}$(indicated by the labels on the arrows). Each polynomial is nonzero, regardless of the parameter values. This follows from evaluation at $x=(1, \mathrm{i})$. Let $z:=t_{1}+\mathrm{i} t_{2}$. 
Proposition 1. If $n=4 m+1$ or $4 m$ with $m \geq 1$ then

$$
\left[p_{n, 1}(1, \mathrm{i}), p_{n, 2}(1, \mathrm{i}), p_{n, 3}(1, \mathrm{i}), p_{n, 4}(1, \mathrm{i})\right]=2^{n-1}[z,-\mathrm{i} z, \bar{z},-\mathrm{i} \bar{z}] ;
$$

if $n=4 m+2$ or $4 m+3$ with $m \geq 0$ then

$$
\left[p_{n, 1}(1, \mathrm{i}), p_{n, 2}(1, \mathrm{i}), p_{n, 3}(1, \mathrm{i}), p_{n, 4}(1, \mathrm{i})\right]=2^{n-1}[\bar{z},-\mathrm{i} \bar{z}, z,-\mathrm{i} z] \text {. }
$$

Proof. The formula is clearly valid for $n=1$. The typical step in the inductive proof is

$$
\left[\begin{array}{l}
p_{n+1,1} \\
p_{n+1,2}
\end{array}\right]=\left[\begin{array}{cc}
1 & \mathrm{i} \\
-\mathrm{i} & 1
\end{array}\right]\left[\begin{array}{c}
c_{1} p_{n, \ell} \\
-\mathrm{i} c_{2} p_{n, \ell}
\end{array}\right]=2\left[\begin{array}{c}
p_{n, \ell} \\
-\mathrm{i} p_{n, \ell}
\end{array}\right]
$$

because $c_{1}+c_{2}=2$. If $n$ is odd then $\ell=3$, otherwise $\ell=1$. The same argument works for $p_{n+1,3}, p_{n+1,4}$.

The types of $p_{2 m+1,1}, p_{2 m+1,2}, p_{2 m+1,3}, p_{2 m+1,4}$ are EE, OO, EE, OO respectively and the types of $p_{2 m, 1}, p_{2 m, 2}, p_{2 m, 3}, p_{2 m, 4}$ are OE, EO, OE, EO respectively.

Proposition 2. For $m \geq 0$

$$
\sigma_{12}^{+}\left[\begin{array}{c}
p_{2 m+1,1} \\
p_{2 m+1,2} \\
p_{2 m+1,3} \\
p_{2 m+1,4}
\end{array}\right]=\left[\begin{array}{c}
p_{2 m+1,1} \\
-p_{2 m+1,2} \\
-p_{2 m+1,3} \\
p_{2 m+1,4}
\end{array}\right], \quad \sigma_{12}^{+}\left[\begin{array}{l}
p_{2 m, 1} \\
p_{2 m, 2} \\
p_{2 m, 3} \\
p_{2 m, 4}
\end{array}\right]=\left[\begin{array}{c}
p_{2 m, 2} \\
p_{2 m, 1} \\
-p_{2 m, 4} \\
-p_{2 m, 3}
\end{array}\right] .
$$

Proof. Using induction one needs to show that the validity of the statements for $2 m-1$ implies the validity for $2 m$, and the validity for $2 m$ implies the validity for $2 m+1$, for each $m \geq 1$. Suppose the statements hold for some $2 m$. The types of $p_{2 m+1, i}$ are easy to verify $(1 \leq i \leq 4)$. Next

$$
\begin{aligned}
\sigma_{12}^{+} p_{2 m+1,1} & =\sigma_{12}^{+}\left(x_{1} p_{2 m, 1}+x_{2} p_{2 m, 2}\right)=x_{2} \sigma_{12}^{+} p_{2 m, 1}+x_{1} \sigma_{12}^{+} p_{2 m, 2} \\
& =x_{2} p_{2 m, 2}+x_{1} p_{2 m, 1}=p_{2 m+1,1}, \\
\sigma_{12}^{+} p_{2 m+1,2} & =\sigma_{12}^{+}\left(x_{1} p_{2 m, 2}-x_{2} p_{2 m, 1}\right)=x_{2} \sigma_{12}^{+} p_{2 m, 2}-x_{1} \sigma_{12}^{+} p_{2 m, 1}=-p_{2 m+1,2},
\end{aligned}
$$

and by similar calculations $\sigma_{12}^{+} p_{2 m+1,3}=-p_{2 m+1,3}$ and $\sigma_{12}^{+} p_{2 m+1,4}=-p_{2 m+1,4}$. Now suppose the statements hold for some $2 m-1$. As before the types of $p_{2 m, i}$ are easy to verify. Consider

$$
\begin{aligned}
\sigma_{12}^{+} p_{2 m, 1} & =\sigma_{12}^{+}\left(\frac{2 m-1+2 k_{-}}{2 m-1} x_{1} p_{2 m-1,3}+\frac{2 m-1-2 k_{-}}{2 m-1} x_{2} p_{2 m-1,4}\right) \\
& =-\frac{2 m-1+2 k_{-}}{2 m-1} x_{2} p_{2 m-1,3}+\frac{2 m-1-2 k_{-}}{2 m-1} x_{1} p_{2 m-1,4}=p_{2 m, 2}, \\
\sigma_{12}^{+} p_{2 m, 3} & =\sigma_{12}^{+}\left(\frac{2 m-1+2 k_{+}}{2 m-1} x_{1} p_{2 m-1,1}+\frac{2 m-1-2 k_{+}}{2 m-1} x_{2} p_{2 m-1,2}\right) \\
& =\frac{2 m-1+2 k_{+}}{2 m-1} x_{2} p_{2 m-1,1}-\frac{2 m-1-2 k_{+}}{2 m-1} x_{1} p_{2 m-1,2}=-p_{2 m, 4} .
\end{aligned}
$$

Since $\left(\sigma_{12}^{+}\right)^{2}=1$ this finishes the inductive proof.

Corollary 2. The polynomials $\left\{p_{2 m+1, i}: 1 \leq i \leq 4\right\}$ are mutually orthogonal.

Proof. These polynomials are eigenfunctions of the (self-adjoint) reflections $\sigma_{1}, \sigma_{12}^{+}$with different pairs of eigenvalues. 
We will use Corollary 1 to evaluate $\mathcal{D}_{i} p_{n, j}$ (with $i=1,2$ and $1 \leq j \leq 4$ ). We intend to show that the expressions appearing in Definition 1 can be interpreted as closed 1-forms $\mathbf{f}$ which are eigenfunctions of $\mathbf{f} \longmapsto \sum_{v \in R_{+}} \kappa(v) \mathbf{f}\left(x \sigma_{v}, t \sigma_{v}\right) \sigma_{v}$. In the present context this is the operator

$$
\rho\left(f_{1}(x, t), f_{2}(x, t)\right):=k_{1}\left(\left(-\sigma_{1}+\sigma_{2}\right) f_{1},\left(\sigma_{1}-\sigma_{2}\right) f_{2}\right)+k_{0}\left(\left(\sigma_{12}^{+}-\sigma_{12}^{-}\right) f_{2},\left(\sigma_{12}^{+}-\sigma_{12}^{-}\right) f_{1}\right) .
$$

It is easy to check that $\left(\sigma_{1}-\sigma_{2}\right) f(x, t)=0=\left(\sigma_{12}^{+}-\sigma_{12}^{-}\right) f(x, t)$ whenever $f \in \mathcal{P}_{V, 2 m+1}$ for $m \geq 0$. For the even degree polynomials $f(x, t)$ one finds $\left(\sigma_{12}^{+}-\sigma_{12}^{-}\right) f=2 \sigma_{12}^{+} f,\left(\sigma_{1}-\sigma_{2}\right) f=-2 f$ if $f$ is of type OE and $\left(\sigma_{1}-\sigma_{2}\right) f=2 f$ if $f$ is of type EO.

Theorem 1. For $m \geq 1$

$$
\begin{array}{ll}
\mathcal{D}_{1} p_{2 m, 1}=2 m \frac{2 m-1+2 k_{-}}{2 m-1} p_{2 m-1,3}, & \mathcal{D}_{2} p_{2 m, 1}=2 m \frac{2 m-1-2 k_{-}}{2 m-1} p_{2 m-1,4}, \\
\mathcal{D}_{1} p_{2 m, 2}=2 m \frac{2 m-1-2 k_{-}}{2 m-1} p_{2 m-1,4}, & \mathcal{D}_{2} p_{2 m, 2}=-2 m \frac{2 m-1+2 k_{-}}{2 m-1} p_{2 m-1,3}, \\
\mathcal{D}_{1} p_{2 m, 3}=2 m \frac{2 m-1+2 k_{+}}{2 m-1} p_{2 m-1,1}, & \mathcal{D}_{2} p_{2 m, 3}=2 m \frac{2 m-1-2 k_{+}}{2 m-1} p_{2 m-1,2}, \\
\mathcal{D}_{1} p_{2 m, 4}=2 m \frac{2 m-1-2 k_{+}}{2 m-1} p_{2 m-1,2}, & \mathcal{D}_{2} p_{2 m, 4}=-2 m \frac{2 m-1+2 k_{+}}{2 m-1} p_{2 m-1,1},
\end{array}
$$

and for $m \geq 1$ (in vector notation)

$$
\begin{aligned}
& {\left[\mathcal{D}_{1}, \mathcal{D}_{2}\right] p_{2 m+1,1}=\left(2 m+1-2 k_{+}\right)\left[p_{2 m, 1}, p_{2 m, 2}\right],} \\
& {\left[\mathcal{D}_{1}, \mathcal{D}_{2}\right] p_{2 m+1,2}=\left(2 m+1+2 k_{+}\right)\left[p_{2 m, 2},-p_{2 m, 1}\right],} \\
& {\left[\mathcal{D}_{1}, \mathcal{D}_{2}\right] p_{2 m+1,3}=\left(2 m+1-2 k_{-}\right)\left[p_{2 m, 3}, p_{2 m, 4}\right],} \\
& {\left[\mathcal{D}_{1}, \mathcal{D}_{2}\right] p_{2 m+1,4}=\left(2 m+1+2 k_{-}\right)\left[p_{2 m, 4},-p_{2 m, 3}\right] .}
\end{aligned}
$$

Proof. Use induction as above. In each case write $p_{n, i}$ in the form $x_{1} f_{1}+x_{2} f_{2}$ with $\mathcal{D}_{2} f_{1}=\mathcal{D}_{1} f_{1}$ and apply Corollary 1. Suppose the statements are true for some $2 m-1$ (direct verification for $m=1)$. Then

$$
p_{2 m, 1}=x_{1}\left(\frac{2 m-1+2 k_{-}}{2 m-1} p_{2 m-1,3}\right)+x_{2}\left(\frac{2 m-1-2 k_{-}}{2 m-1} p_{2 m-1,4}\right)
$$

and

$$
\begin{aligned}
\mathcal{D}_{2}\left(\frac{2 m-1+2 k_{-}}{2 m-1} p_{2 m-1,3}\right) & =\frac{\left(2 m-1+2 k_{-}\right)\left(2 m-1-2 k_{-}\right)}{2 m-1} p_{2 m-2,4} \\
& =\mathcal{D}_{1}\left(\frac{2 m-1-2 k_{-}}{2 m-1} p_{2 m-1,4}\right)
\end{aligned}
$$

by the inductive hypothesis. Since $\rho p_{2 m-1, i}=0$ for $1 \leq i \leq 4$ Corollary 1 shows that $\mathcal{D}_{1} p_{2 m, 1}=$ $2 m\left(\frac{2 m-1+2 k_{-}}{2 m-1} p_{2 m-1,3}\right)$ and $\mathcal{D}_{2} p_{2 m, 1}$ has the stated value. Also

$$
p_{2 m, 1}=x_{1}\left(\frac{2 m-1-2 k_{+}}{2 m-1} p_{2 m-1,2}\right)+x_{2}\left(-\frac{2 m-1+2 k_{+}}{2 m-1} p_{2 m-1,1}\right),
$$

and

$$
\begin{aligned}
\mathcal{D}_{2}\left(\frac{2 m-1-2 k_{+}}{2 m-1} p_{2 m-1,2}\right) & =-\frac{\left(2 m-1+2 k_{-}\right)\left(2 m-1-2 k_{+}\right)}{2 m-1} p_{2 m-2,1} \\
& =\mathcal{D}_{1}\left(-\frac{2 m-1+2 k_{+}}{2 m-1} p_{2 m-1,2}\right)
\end{aligned}
$$


and so $\mathcal{D}_{2} p_{2 m, 4}=2 m\left(-\frac{2 m-1+2 k_{+}}{2 m-1} p_{2 m-1,1}\right)$. The other statements for $p_{2 m, i}$ have similar proofs. Now assume the statements are true for some $2 \mathrm{~m}$. The desired relations follow from Corollary 1 and the following

$$
\begin{aligned}
& \rho\left(p_{2 m, 1}, p_{2 m, 2}\right)=2 k_{0}\left(\sigma_{12}^{+} p_{2 m, 2}, \sigma_{12}^{+} p_{2 m, 1}\right)+2 k_{1}\left(p_{2 m, 1}, p_{2 m, 2}\right) \\
& =\left(2 k_{0}+2 k_{1}\right)\left(p_{2 m, 1}, p_{2 m, 2}\right), \quad \lambda_{\kappa}=2 k_{+} ; \\
& \begin{aligned}
\rho\left(p_{2 m, 2},-p_{2 m, 1}\right)= & 2 k_{0}\left(-\sigma_{12}^{+} p_{2 m, 1}, \sigma_{12}^{+} p_{2 m, 2}\right)-2 k_{1}\left(p_{2 m, 2},-p_{2 m, 1}\right) \\
= & \left(-2 k_{0}-2 k_{1}\right)\left(p_{2 m, 2},-p_{2 m, 1}\right), \quad \lambda_{\kappa}=-2 k_{+} ; \\
\rho\left(p_{2 m, 3}, p_{2 m, 4}\right)= & 2 k_{0}\left(\sigma_{12}^{+} p_{2 m, 4}, \sigma_{12}^{+} p_{2 m, 3}\right)+2 k_{1}\left(p_{2 m, 3}, p_{2 m, 4}\right) \\
= & \left(-2 k_{0}+2 k_{1}\right)\left(p_{2 m, 3}, p_{2 m, 4}\right), \quad \lambda_{\kappa}=2 k_{-} ; \\
\rho\left(p_{2 m, 4},-p_{2 m, 3}\right)= & 2 k_{0}\left(-\sigma_{12}^{+} p_{2 m, 3}, \sigma_{12}^{+} p_{2 m, 4}\right)-2 k_{1}\left(p_{2 m, 4},-p_{2 m, 3}\right) \\
= & \left(2 k_{0}-2 k_{1}\right)\left(p_{2 m, 4},-p_{2 m, 3}\right), \quad \lambda_{\kappa}=-2 k_{-} .
\end{aligned}
\end{aligned}
$$

The closed 1-form condition is shown by using the inductive hypothesis. A typical calculation is

$$
p_{2 m+1,2}=x_{1} p_{2 m, 2}-x_{2} p_{2 m, 1}, \quad \mathcal{D}_{2} p_{2 m, 2}=-2 m \frac{2 m-1+2 k_{-}}{2 m-1} p_{2 m-1,3}=-\mathcal{D}_{1} p_{2 m, 1} .
$$

This completes the inductive proof.

Corollary 3. Suppose $n \geq 1$ and $1 \leq i \leq 4$ then $\Delta_{\kappa} p_{n, i}=0$.

Proof. By the above formulae $\mathcal{D}_{1}^{2} p_{n, i}=-\mathcal{D}_{2}^{2} p_{n, i}$ in each case. As a typical calculation

$$
\begin{aligned}
\mathcal{D}_{1}^{2} p_{2 m, 1} & =\mathcal{D}_{1}\left(2 m \frac{2 m-1+2 k_{-}}{2 m-1} p_{2 m-1,3}\right) \\
& =\frac{2 m}{2 m-1}\left(2 m-1+2 k_{-}\right)\left(2 m+1-2 k_{-}\right) p_{2 m-2,3}, \\
\mathcal{D}_{2}^{2} p_{2 m, 1} & =\mathcal{D}_{2}\left(2 m \frac{2 m-1-2 k_{-}}{2 m-1} p_{2 m-1,4}\right) \\
& =\frac{2 m}{2 m-1}\left(2 m+1-2 k_{-}\right)\left(2 m+1+2 k_{-}\right)\left(-p_{2 m-2,3}\right) .
\end{aligned}
$$

It suffices to check the even degree cases because $\Delta_{\kappa} f=0$ implies $\Delta_{\kappa} \mathcal{D}_{1} f=0$.

Because $p_{2 m, 1}$ and $p_{2 m, 3}$ are both type $\mathrm{OE}$ one can use an appropriate self-adjoint operator to prove orthogonality. Indeed let $\mathcal{U}_{12}:=\sigma_{12}^{+}\left(x_{2} \mathcal{D}_{1}-x_{1} \mathcal{D}_{2}\right)$, which is self-adjoint for the form $\langle\cdot, \cdot\rangle_{\tau}$.

Proposition 3. Suppose $m \geq 1$ and $1 \leq i \leq 4$ then $\mathcal{U}_{12} p_{2 m, i}=2 m \varepsilon_{i} p_{2 m, i}$ where $\varepsilon_{1}=\varepsilon_{4}=-1$ and $\varepsilon_{2}=\varepsilon_{3}=1$.

Proof. This is a simple verification. For example

$$
\begin{aligned}
\left(x_{2} \mathcal{D}_{1}-x_{1} \mathcal{D}_{2}\right) p_{2 m, 4} & =\frac{2 m}{2 m-1}\left(x_{2}\left(2 m-1-2 k_{+}\right) p_{2 m-1,3}+x_{1}\left(2 m-1+2 k_{+}\right) p_{2 m-1,4}\right) \\
& =2 m p_{2 m, 3},
\end{aligned}
$$

and $\sigma_{12}^{+} p_{2 m, 3}=-p_{2 m, 4}$.

Corollary 4. For $m \geq 1$ the polynomials $\left\{p_{2 m, i}: 1 \leq i \leq 4\right\}$ are mutually orthogonal.

Proof. These polynomials are eigenfunctions of the self-adjoint operators $\sigma_{1}$ and $\mathcal{U}_{12}$ with different pairs of eigenvalues. 
Proposition 4. For any $k_{0}, k_{1}$ and $n \geq 1$ the polynomials $\left\{p_{n, i}: 1 \leq i \leq 4\right\}$ form a basis for $\mathcal{H}_{V, \kappa, n}$.

Proof. The fact that $p_{n, i} \neq 0$ for all $n, i$ (Proposition 1 ) and the eigenvector properties from Propositions 2 and 3 imply linear independence for each set $\left\{p_{n, i}: 1 \leq i \leq 4\right\}$.

We introduce the notation $\nu(f):=\langle f, f\rangle_{\tau}$ (without implying that the form is positive). To evaluate $\nu\left(p_{n, i}\right)$ we will use induction and the following simple fact: suppose $f=x_{1} f_{1}+x_{2} f_{2}$ and $\mathcal{D}_{i} f=\alpha f_{i}$ for $i=1,2$ and some $\alpha \in \mathbb{Q}\left[k_{0}, k_{1}\right]$ then

$$
\nu(f)=\left\langle x_{1} f_{1}, f\right\rangle_{\tau}+\left\langle x_{2} f_{2}, f\right\rangle_{\tau}=\left\langle f_{1}, \mathcal{D}_{1} f\right\rangle_{\tau}+\left\langle f_{2}, \mathcal{D}_{2} f\right\rangle_{\tau}=\alpha\left(\nu\left(f_{1}\right)+\nu\left(f_{2}\right)\right) .
$$

By use of the various formulae we obtain

$$
\begin{aligned}
& \nu\left(p_{2 m, 1}\right)=\nu\left(p_{2 m, 2}\right)=2 m\left(\left(\frac{2 m-1+2 k_{-}}{2 m-1}\right)^{2} \nu\left(p_{2 m-1,3}\right)+\left(\frac{2 m-1-2 k_{-}}{2 m-1}\right)^{2} \nu\left(p_{2 m-1,4}\right)\right), \\
& \nu\left(p_{2 m, 3}\right)=\nu\left(p_{2 m, 4}\right)=2 m\left(\left(\frac{2 m-1+2 k_{+}}{2 m-1}\right)^{2} \nu\left(p_{2 m-1,1}\right)+\left(\frac{2 m-1-2 k_{+}}{2 m-1}\right)^{2} \nu\left(p_{2 m-1,2}\right)\right),
\end{aligned}
$$

and

$$
\begin{array}{ll}
\nu\left(p_{2 m+1,1}\right)=2\left(2 m+1-2 k_{+}\right) \nu\left(p_{2 m, 1}\right), & \nu\left(p_{2 m+1,2}\right)=2\left(2 m+1+2 k_{+}\right) \nu\left(p_{2 m, 1}\right), \\
\nu\left(p_{2 m+1,3}\right)=2\left(2 m+1-2 k_{-}\right) \nu\left(p_{2 m, 3}\right), & \nu\left(p_{2 m+1,4}\right)=2\left(2 m+1+2 k_{-}\right) \nu\left(p_{2 m, 3}\right) .
\end{array}
$$

From these relations it follows that

$$
\begin{aligned}
& \nu\left(p_{2 m+2,1}\right)=8(m+1) \frac{\left(2 m+1-2 k_{-}\right)\left(2 m+1+2 k_{-}\right)}{2 m+1} \nu\left(p_{2 m, 3}\right), \\
& \nu\left(p_{2 m+2,3}\right)=8(m+1) \frac{\left(2 m+1-2 k_{+}\right)\left(2 m+1+2 k_{+}\right)}{2 m+1} \nu\left(p_{2 m, 1}\right) .
\end{aligned}
$$

It is now a case-by-case verification for an inductive proof. Define a Pochhammer-type function (referred to as " $\Pi$ " in the sequel) for notational convenience

$$
\Pi\left(a, b, m, \varepsilon_{1} \varepsilon_{2} \varepsilon_{3} \varepsilon_{4}\right):=\frac{\left(\frac{1}{4}+\frac{a}{2}\right)_{m+\varepsilon_{1}}\left(\frac{1}{4}-\frac{a}{2}\right)_{m+\varepsilon_{2}}\left(\frac{3}{4}+\frac{b}{2}\right)_{m+\varepsilon_{3}}\left(\frac{3}{4}-\frac{b}{2}\right)_{m+\varepsilon_{4}}}{\left(\frac{1}{4}\right)_{m+\varepsilon_{1}}\left(\frac{1}{4}\right)_{m+\varepsilon_{2}}\left(\frac{3}{4}\right)_{m+\varepsilon_{3}}\left(\frac{3}{4}\right)_{m+\varepsilon_{4}}},
$$

where $m \geq 0$ and $\varepsilon_{1} \varepsilon_{2} \varepsilon_{3} \varepsilon_{4}$ is a list with $\varepsilon_{i}=0$ or 1 . To avoid repetitions of the factor $2^{n} n$ ! let

$$
\nu^{\prime}\left(p_{n, i}\right):=\nu\left(p_{n, i}\right) /\left(2^{n} n !\right) .
$$

Then

$$
\begin{aligned}
& \nu^{\prime}\left(p_{4 m, 1}\right)=\nu^{\prime}\left(p_{4 m, 2}\right)=\Pi\left(k_{+}, k_{-}, m, 0000\right), \\
& \nu^{\prime}\left(p_{4 m, 3}\right)=\nu^{\prime}\left(p_{4 m, 4}\right)=\Pi\left(k_{-}, k_{+}, m, 0000\right), \\
& \nu^{\prime}\left(p_{4 m+2,1}\right)=\nu^{\prime}\left(p_{4 m+2,2}\right)=\Pi\left(k_{-}, k_{+}, m, 1100\right), \\
& \nu^{\prime}\left(p_{4 m+2,3}\right)=\nu^{\prime}\left(p_{4 m+2,4}\right)=\Pi\left(k_{+}, k_{-}, m, 1100\right),
\end{aligned}
$$

and

$$
\begin{array}{ll}
\nu^{\prime}\left(p_{4 m+1,1}\right)=\Pi\left(k_{+}, k_{-}, m, 0100\right), & \nu^{\prime}\left(p_{4 m+1,2}\right)=\Pi\left(k_{+}, k_{-}, m, 1000\right), \\
\nu^{\prime}\left(p_{4 m+1,3}\right)=\Pi\left(k_{-}, k_{+}, m, 0100\right), & \nu^{\prime}\left(p_{4 m+1,4}\right)=\Pi\left(k_{-}, k_{+}, m, 1000\right),
\end{array}
$$




$$
\begin{array}{ll}
\nu^{\prime}\left(p_{4 m+3,1}\right)=\Pi\left(k_{-}, k_{+}, m, 1101\right), & \nu^{\prime}\left(p_{4 m+3,2}\right)=\Pi\left(k_{-}, k_{+}, m, 1110\right), \\
\nu^{\prime}\left(p_{4 m+3,3}\right)=\Pi\left(k_{+}, k_{-}, m, 1101\right), & \nu^{\prime}\left(p_{4 m+3,4}\right)=\Pi\left(k_{+}, k_{-}, m, 1110\right) .
\end{array}
$$

These formulae, together with the harmonic decomposition of polynomials, prove that $\langle\cdot, \cdot\rangle_{\tau}$ is positive-definite for $-\frac{1}{2}<k_{+}, k_{-}<\frac{1}{2}$ (equivalently $-\frac{1}{2}< \pm k_{0} \pm k_{1}<\frac{1}{2}$ ) (this is a special case of $[6$, Proposition 4.4]).

These norm results are used to analyze the representation of the rational Cherednik algebra $\mathcal{A}_{\kappa}$ on $\mathcal{P}_{V}$ for arbitrary parameter values. For fixed $k_{0}, k_{1}$ the radical in $\mathcal{P}_{V}$ is the subspace

$$
\operatorname{rad}_{V}\left(k_{0}, k_{1}\right):=\left\{p:\langle p, q\rangle_{\tau}=0 \forall q \in \mathcal{P}_{V}\right\} .
$$

The radical is an $\mathcal{A}_{\kappa}$-module and the representation is called unitary if the form $\langle\cdot, \cdot\rangle_{\tau}$ is positivedefinite on $\mathcal{P}_{V} / \operatorname{rad}_{V}\left(k_{0}, k_{1}\right)$. By Proposition $4\left\{|x|^{2 m} p_{n, i}\right\}$ (with $m, n \in \mathbb{N}_{0}, 1 \leq i \leq 4$, except $1 \leq i \leq 2$ when $n=0$ ) is a basis for $\mathcal{P}_{V}$ for any parameter values (see formula (5)).

Proposition 5. For fixed $k_{0}, k_{1}$ the set $\left\{|x|^{2 m} p_{n, i}: \nu\left(p_{n, i}\right)=0\right\}$ is a basis for $\operatorname{rad}_{V}\left(k_{0}, k_{1}\right)$.

Proof. Suppose $p=\sum_{m, n, i} a_{m, n, i}|x|^{2 m} p_{n, i} \in \operatorname{rad}_{V}\left(k_{0}, k_{1}\right)$ then $\left\langle p,|x|^{2 \ell} p_{k, j}\right\rangle_{\tau}=a_{\ell, k, j} 4^{\ell} \ell !(k+1)_{\ell} \nu\left(p_{k, j}\right)$ by (6), thus $a_{\ell, k, j} \neq 0$ implies $\nu\left(p_{k, j}\right)=0$.

For a given value $k_{+}=\frac{1}{2}+m_{+}$or $k_{-}=\frac{1}{2}+m_{-}$(or both) with $m_{+}, m_{-} \in \mathbb{Z}$ the polynomials with $\nu\left(p_{k, j}\right)=0$ can be determined from the above norm formulae. For example let $k_{+}=-\frac{1}{2}$ then $\operatorname{rad}_{V}\left(k_{0},-\frac{1}{2}-k_{0}\right)$ contains $p_{n, i}$ for $(n, i)$ in the list $(1,2),(n, 1),(n, 2)(n \geq 2$ and $n \equiv$ $0,1 \bmod 4),(n, 3),(n, 4)(n \geq 2$ and $n \equiv 2,3 \bmod 4)$. In particular each point on the boundary of the square $-\frac{1}{2}<k_{0} \pm k_{1}<\frac{1}{2}$ corresponds to a unitary representation of $\mathcal{A}_{\kappa}$.

\section{The reproducing kernel}

In the $\tau=1$ setting there is a ("Dunkl") kernel $E(x, y)$ which satisfies $\mathcal{D}_{i}^{(x)} E(x, y)=y_{i} E(x, y)$, $E(x, y)=E(y, x)$ and $\langle E(\cdot, y), p\rangle_{1}=p(y)$ for any polynomial $p$. We show there exists such a function in this $B_{2}$-setting which is real-analytic in its arguments, provided $\frac{1}{2} \pm k_{0} \pm k_{1} \notin \mathbb{Z}$. This kernel takes values in $V \otimes V$; for notational convenience we will use expressions of the form $\sum_{i=1}^{2} \sum_{j=1}^{2} f_{i j}(x, y) s_{i} t_{j}$ where each $f_{i j}(x, y)$ is a polynomial in $x_{1}, x_{2}, y_{1}, y_{2}$ (technically, we should write $s_{i} \otimes t_{j}$ for the basis elements in $\left.V \otimes V\right)$. For a polynomial $f(x)=f_{1}(x) t_{1}+f_{2}(x) t_{2}$ let $f(y)^{*}$ denote $f_{1}(y) s_{1}+f_{2}(y) s_{2}$. The kernel $E$ is defined as a sum of terms like $\frac{1}{\nu\left(p_{n, i}\right)} p_{n, i}(y)^{*} p_{n, i}(x)$; by the orthogonality relations (for $m, n=0,1,2, \ldots$ and $1 \leq i, j \leq 4$ )

$$
\frac{1}{\nu\left(p_{n, i}\right)} p_{n, i}(y)^{*}\left\langle p_{n, i}, p_{m, j}\right\rangle_{\tau}=\delta_{m n} \delta_{i j} p_{n, i}(y)^{*}
$$

By formulae (4) and (6)

$$
\left\langle|x|^{2 a} p_{n, i}(x),|x|^{2 b} p_{m, j}(x)\right\rangle_{\tau}=\delta_{n m} \delta_{a b} \delta_{i j} 4^{a} a !(n+1)_{a} \nu\left(p_{n, i}\right) .
$$

We will find upper bounds on $\left\{p_{n, i}\right\}$ and lower bounds on $\left\{\nu\left(p_{n, i}\right)\right\}$ in order to establish convergence properties of $E(x, y)$. For $u \in \mathbb{R}$ set

$$
d(u):=\min _{m \in \mathbb{Z}}\left|u+\frac{1}{2}+m\right| .
$$

The condition that $\nu\left(p_{n, i}\right) \neq 0$ for all $n \geq 1,1 \leq i \leq 4$ is equivalent to $d\left(k_{+}\right) d\left(k_{-}\right)>0$, since each factor in the numerator of $\Pi$ is of the form $\frac{1}{4}+\frac{1}{2}\left( \pm k_{0} \pm k_{1}\right)+m$ or $\frac{3}{4}+\frac{1}{2}\left( \pm k_{0} \pm k_{1}\right)+m$ for $m=0,1,2, \ldots$. 
Definition 2. Let $P_{0}(x, y):=s_{1} t_{1}+s_{2} t_{2}$ and for $n \geq 1$ let

$$
P_{n}(x, y):=\sum_{i=1}^{4} \frac{1}{\nu\left(p_{n, i}\right)} p_{n, i}(y)^{*} p_{n, i}(x) .
$$

For $n \geq 0$ let

$$
E_{n}(x, y):=\sum_{0 \leq m \leq n / 2} \frac{\left(x_{1}^{2}+x_{2}^{2}\right)^{m}\left(y_{1}^{2}+y_{2}^{2}\right)^{m}}{4^{m} m !(n-2 m+1)_{m}} P_{n-2 m}(x, y) .
$$

Proposition 6. If $k_{0}, k_{1}$ are generic or $d\left(k_{+}\right) d\left(k_{-}\right)>0$, and $n \geq 1$ then $\left\langle E_{n}(\cdot, y), f\right\rangle_{\tau}=f(y)^{*}$ for each $f \in \mathcal{P}_{V, n}$, and $\mathcal{D}_{i}^{(x)} E_{n}(x, y)=y_{i} E_{n-1}(x, y)$ for $i=1,2$.

Proof. By hypothesis on $k_{0}, k_{1}$ the polynomial $E_{n}(x, y)$ exists, and is a rational function of $k_{0}, k_{1}$. The reproducing property is a consequence of the orthogonal decomposition $\mathcal{P}_{V, n}=$ $\sum_{0 \leq m \leq n / 2} \oplus|x|^{2 m} \mathcal{H}_{V, \kappa, n-2 m}$. For the second part, suppose $f \in \mathcal{P}_{V, n-1}$, then

$$
\left\langle\mathcal{D}_{i}^{(x)} E_{n}(x, y), f(x)\right\rangle_{\tau}=\left\langle E_{n}(x, y), x_{i} f(x)\right\rangle_{\tau}=y_{i} f(y)^{*}=y_{i}\left\langle E_{n-1}(x, y), f(x)\right\rangle_{\tau} .
$$

If $-\frac{1}{2}< \pm k_{0} \pm k_{1}<\frac{1}{2}$ the bilinear form is positive-definite which implies $\mathcal{D}_{i}^{(x)} E_{n}(x, y)=$ $y_{i} E_{n-1}(x, y)$. This is an algebraic (rational) relation which holds on an open set of its arguments and is thus valid for all $k_{0}, k_{1}$ except for the poles of $E_{n}$.

Write $E_{n}(x, y)=\sum_{i, j=1}^{2} E_{n, i j}(x, y) s_{i} t_{j}$ then the equivariance relation becomes $E_{n, i j}(x w, y w)=$ $\left(w^{-1} E_{n, . .}(x, y) w\right)_{i j}$ for each $w \in W\left(B_{2}\right)$. Next we show that $\sum_{n=0}^{\infty} E_{n}(x, y)$ converges to a realentire function in $x, y$. For $p(x, t)=p_{1}(x) t_{1}+p_{2}(x) t_{2} \in \mathcal{P}_{V}$ let $\beta(p)(x)=p_{1}(x)^{2}+p_{2}(x)^{2}$.

Lemma 2. Suppose $m \geq 0$ and $i=1$ or 3 then

$$
\beta\left(p_{2 m+1, i}\right)(x)+\beta\left(p_{2 m+1, i+1}\right)(x)=\left(x_{1}^{2}+x_{2}^{2}\right)\left(\beta\left(p_{2 m, i}\right)(x)+\beta\left(p_{2 m, i+1}\right)(x)\right) .
$$

Proof. By Definition $1 p_{2 m+1, i}=x_{1} p_{2 m, i}+x_{2} p_{2 m, i+1}$ and $p_{2 m+1, i+1}=-x_{2} p_{2 m, i}+x_{1} p_{2 m, i+1}$. The result follows from direct calculation using these formulae.

Lemma 3. Suppose $m \geq 1$ then

$$
\begin{aligned}
& \beta\left(p_{2 m, 1}\right)(x)+\beta\left(p_{2 m, 2}\right)(x) \\
& =\left(x_{1}^{2}+x_{2}^{2}\right)\left(\left(\frac{2 m-1+2 k_{-}}{2 m-1}\right)^{2} \beta\left(p_{2 m-1,3}\right)(x)+\left(\frac{2 m-1-2 k_{-}}{2 m-1}\right)^{2} \beta\left(p_{2 m-1,4}\right)(x)\right), \\
& \beta\left(p_{2 m, 3}\right)(x)+\beta\left(p_{2 m, 4}\right)(x) \\
& =\left(x_{1}^{2}+x_{2}^{2}\right)\left(\left(\frac{2 m-1+2 k_{+}}{2 m-1}\right)^{2} \beta\left(p_{2 m-1,1}\right)(x)+\left(\frac{2 m-1-2 k_{+}}{2 m-1}\right)^{2} \beta\left(p_{2 m-1,2}\right)(x)\right) .
\end{aligned}
$$

Proof. This follows similarly as the previous argument.

Proposition 7. Suppose $m \geq 0$ and $i=1$ or 3 then

$$
\begin{aligned}
& \beta\left(p_{2 m, i}\right)(x)+\beta\left(p_{2 m, i+1}\right)(x) \leq 2\left(\frac{\left(1 / 2+\left|k_{0}\right|+\left|k_{1}\right|\right)_{m}}{(1 / 2)_{m}}\right)^{2}\left(x_{1}^{2}+x_{2}^{2}\right)^{2 m}, \\
& \beta\left(p_{2 m+1, i}\right)(x)+\beta\left(p_{2 m+1, i+1}\right)(x) \leq 2\left(\frac{\left(1 / 2+\left|k_{0}\right|+\left|k_{1}\right|\right)_{m}}{(1 / 2)_{m}}\right)^{2}\left(x_{1}^{2}+x_{2}^{2}\right)^{2 m+1} .
\end{aligned}
$$


Proof. Use induction, the lemmas, and the inequality $\left(\frac{2 m-1 \pm 2 k_{0} \pm 2 k_{1}}{2 m-1}\right)^{2} \leq\left(\frac{m-1 / 2+\left|k_{0}\right|+\left|k_{1}\right|}{m-1 / 2}\right)^{2}$. The beginning step is $\beta\left(p_{1, i}\right)(x)+\beta\left(p_{1, i+1}\right)(x)=2\left(x_{1}^{2}+x_{2}^{2}\right)$.

By Stirling's formula

$$
\begin{aligned}
\frac{\left(1 / 2+\left|k_{0}\right|+\left|k_{1}\right|\right)_{m}}{(1 / 2)_{m}} & =\frac{\Gamma(1 / 2)}{\Gamma\left(1 / 2+\left|k_{0}\right|+\left|k_{1}\right|\right)} \frac{\Gamma\left(1 / 2+\left|k_{0}\right|+\left|k_{1}\right|+m\right)}{\Gamma(1 / 2+m)} \\
& \sim \frac{\Gamma(1 / 2)}{\Gamma\left(1 / 2+\left|k_{0}\right|+\left|k_{1}\right|\right)} m^{\left|k_{0}\right|+\left|k_{1}\right|}
\end{aligned}
$$

as $m \rightarrow \infty$. For the purpose of analyzing lower bounds on $\left|\nu^{\prime}\left(p_{n, i}\right)\right|$ we consider an infinite product.

Lemma 4. Suppose $u>0$ then the infinite product

$$
\omega(u ; z):=\prod_{n=0}^{\infty}\left(1-\frac{z^{2}}{(u+n)^{2}}\right)
$$

converges to an entire function of $z \in \mathbb{C}$, satisfies

$$
\omega(u ; z)=\frac{\Gamma(u)^{2}}{\Gamma(u+z) \Gamma(u-z)},
$$

and has simple zeros at $\pm(u+n), n=0,1,2, \ldots$

Proof. The product converges to an entire function by the comparison test: $\sum_{n=1}^{\infty} \frac{|z|^{2}}{(u+n)^{2}}<\infty$ (this means that the partial products converge to a nonzero limit, unless one of the factors vanishes). Suppose that $|z|<u$ then $\operatorname{Re}(u \pm z) \geq u-|z|>0$ and

$$
\begin{aligned}
& \prod_{n=0}^{\infty}\left(1-\frac{z^{2}}{(u+n)^{2}}\right)=\lim _{m \rightarrow \infty} \frac{(u+z)_{m}(u-z)_{m}}{(u)_{m}^{2}} \\
& \quad=\frac{\Gamma(u)^{2}}{\Gamma(u+z) \Gamma(u-z)} \lim _{m \rightarrow \infty} \frac{\Gamma(u+z+m) \Gamma(u-z+m)}{\Gamma(u+m)^{2}}=\frac{\Gamma(u)^{2}}{\Gamma(u+z) \Gamma(u-z)},
\end{aligned}
$$

by Stirling's formula. The entire function $\omega(u ; \cdot)$ agrees with the latter expression (in $\Gamma$ ) on an open set in $\mathbb{C}$, hence for all $z$.

\section{Corollary 5.}

1. If $n \equiv 0,1 \bmod 4$ and $i=1,2$ or $n \equiv 2,3 \bmod 4$ and $i=3,4$ then

$$
\lim _{n \rightarrow \infty} \nu^{\prime}\left(p_{n, i}\right)=\omega\left(\frac{1}{4} ; \frac{k_{+}}{2}\right) \omega\left(\frac{3}{4} ; \frac{k_{-}}{2}\right) .
$$

2. If $n \equiv 0,1 \bmod 4$ and $i=3,4$ or $n \equiv 2,3 \bmod 4$ and $i=1,2$ then

$$
\lim _{n \rightarrow \infty} \nu^{\prime}\left(p_{n, i}\right)=\omega\left(\frac{1}{4} ; \frac{k_{-}}{2}\right) \omega\left(\frac{3}{4} ; \frac{k_{+}}{2}\right) .
$$

Proof. The statements follow from formulae (8), (9) and (10).

For $a \in \mathbb{R}$ with $a+\frac{1}{2} \notin \mathbb{Z}$ let $((a))$ denote the nearest integer to $a$. 
Lemma 5. For each $\left(n_{1}, n_{2}\right) \in \mathbb{Z}^{2}$ there is a constant $C\left(n_{1}, n_{2}\right)$ such that $\left(\left(k_{+}\right)\right)=n_{1}$ and $\left(\left(k_{-}\right)\right)=n_{2}$ implies $\left|\nu^{\prime}\left(p_{n, i}\right)\right| \geq C\left(n_{1}, n_{2}\right) d\left(k_{+}\right) d\left(k_{-}\right)$.

Proof. It suffices to consider Case 1 in the corollary (interchange $k_{+}$and $k_{-}$to get Case 2). The limit function has zeros at $k_{+}= \pm \frac{4 m+1}{2}$ and $k_{-}= \pm \frac{4 m+3}{2}$ for $m=0,1,2, \ldots$ For each $n_{1} \neq 0$ and $n_{2} \neq 0$ there are unique nearest zeros $k_{+}=z_{1}$ and $k_{-}=z_{2}$ respectively; for example if $n_{1}$ is odd and $n_{1} \geq 1$ then $z_{1}=n_{1}-\frac{1}{2}$; and if $n_{2}$ is even and $n_{2} \leq-2$ then $z_{2}=n_{2}+\frac{1}{2}$. Consider the entire function

$$
f\left(k_{+}, k_{-}\right)=\frac{1}{\left(z_{1}-k_{+}\right)\left(z_{2}-k_{-}\right)} \omega\left(\frac{1}{4} ; \frac{k_{+}}{2}\right) \omega\left(\frac{3}{4} ; \frac{k_{-}}{2}\right) .
$$

If $n_{1}=0$ then replace the factor $\left(z_{1}-k_{+}\right)$by $\left(\frac{1}{4}-k_{+}^{2}\right)$, and if $n_{2}=0$ replace $\left(z_{2}-k_{-}\right)$by 1 . In each of these cases the quotient is an entire function with no zeros in $\left|n_{1}-k_{+}\right| \leq \frac{1}{2}$ and $\left|n_{2}-k_{-}\right| \leq \frac{1}{2}$. Thus there is a lower bound $C_{1}$ in absolute value for all the partial products of $f$, valid for all $k_{+}, k_{-}$in this region. The expressions for $\nu^{\prime}\left(p_{n, i}\right)$ (see formulae (8), (9) and (10))

involve terms in $\left\{\varepsilon_{i}\right\}$ but these do not affect the convergence properties (and note $0 \leq \sum_{i=1}^{4} \varepsilon_{i} \leq 3$ in each case). Since $\left|z_{1}-k_{+}\right| \geq d\left(k_{+}\right)$and $\left|z_{2}-k_{-}\right| \geq d\left(k_{-}\right)$we find that the partial products of $\left(z_{1}-k_{+}\right)\left(z_{2}-k_{-}\right) f\left(k_{+}, k_{-}\right)$, that is, the values of $\nu\left(p_{n, i}\right) /\left(2^{n} n !\right)$, are bounded below by $d\left(k_{+}\right) d\left(k_{-}\right) C_{1}$ in absolute value. In case $n_{1}=0$ the factor $\left(\frac{1}{4}-k_{+}^{2}\right)=d\left(k_{+}\right)\left(1-d\left(k_{+}\right)\right) \geq \frac{1}{2} d\left(k_{+}\right)$ (and in the more trivial case $n_{2}=0$ one has $1>\frac{1}{2} \geq d\left(k_{-}\right)$).

Theorem 2. For a fixed $k_{0}, k_{1} \in \mathbb{R}$ satisfying $d\left(k_{+}\right) d\left(k_{-}\right)>0$ the series $E(x, y):=\sum_{n=0}^{\infty} E_{n}(x, y)$ converges absolutely and uniformly on $\left\{(x, y) \in \mathbb{R}^{4}:|x|^{2}+|y|^{2} \leq R^{2}\right\}$ for any $R>0$.

Proof. We have shown there is a constant $C^{\prime}>0$ such that

$$
\left|P_{n}(x, y)\right| \leq C^{\prime} \frac{|x|^{n}|y|^{n}}{2^{n} n !}\left(\frac{n}{2}\right)^{\left|k_{0}\right|+\left|k_{1}\right|},
$$

and thus the series

$$
E(x, y)=\sum_{n=0}^{\infty} \sum_{0 \leq m \leq n / 2} \frac{|x|^{2 m}|y|^{2 m}}{4^{m} m !(n-2 m+1)_{m}} P_{n-2 m}(x, y)=\sum_{l=0}^{\infty} P_{l}(x, y) \sum_{m=0}^{\infty} \frac{|x|^{2 m}|y|^{2 m}}{4^{m} m !(l+1)_{m}}
$$

converges uniformly for $|x|^{2}+|y|^{2}<R^{2}$.

Corollary 6. Suppose $f \in \mathcal{P}_{V}$ then $\langle E(\cdot, y), f\rangle_{\tau}=f(y)^{*} ;$ also $\mathcal{D}_{i}^{(x)} E(x, y)=y_{i} E(x, y)$ for $i=1,2$.

As in the scalar $(\tau=1)$ theory the function $E(x, y)$ can be used to define a generalized Fourier transform.

\section{The Gaussian-type weight function}

In this section we use vector notation for $\mathcal{P}_{V}: f(x)=\left(f_{1}(x), f_{2}(x)\right)$ for the previous $f_{1}(x) t_{1}+$ $f_{2}(x) t_{2}$. The action of $W$ is written as $(w f)(x)=f(x w) w^{-1}$. We propose to construct a $2 \times 2$ positive-definite matrix function $K(x)$ on $\mathbb{R}^{2}$ such that

$$
\langle f, g\rangle_{G}=\int_{\mathbb{R}^{2}} f(x) K(x) g(x)^{T} e^{-|x|^{2} / 2} d x, \quad \forall f, g \in \mathcal{P}_{V},
$$


and with the restriction $\left|k_{0} \pm k_{1}\right|<\frac{1}{2}$; the need for this was demonstrated in the previous section. The two necessary algebraic conditions are (for all $f, g \in \mathcal{P}_{V}$ )

$$
\begin{aligned}
& \langle f, g\rangle_{G}=\langle w f, w g\rangle_{G}, \quad w \in W, \\
& \left\langle\left(x_{i}-\mathcal{D}_{i}\right) f, g\right\rangle_{G}=\left\langle f, \mathcal{D}_{i} g\right\rangle_{G}, \quad i=1,2 .
\end{aligned}
$$

We will assume $K$ is differentiable on $\Omega:=\left\{x \in \mathbb{R}^{2}: x_{1} x_{2}\left(x_{1}^{2}-x_{2}^{2}\right) \neq 0\right\}$. The integral formula is defined if $K$ is integrable, but for the purpose of dealing with the singularities implicit in $\mathcal{D}_{i}$ we introduce the region

$$
\Omega_{\varepsilon}:=\left\{x: \min \left(\left|x_{1}\right|,\left|x_{2}\right|,\left|x_{1}-x_{2}\right|,\left|x_{1}+x_{2}\right|\right) \geq \varepsilon\right\}
$$

for $\varepsilon>0$. The fundamental region of $W$ corresponding to $R_{+}$is $\mathcal{C}_{0}=\left\{x: 0<x_{2}<x_{1}\right\}$.

Condition (11) implies, for each $w \in W, f, g \in \mathcal{P}_{V}$ that

$$
\begin{aligned}
\int_{\mathbb{R}^{2}} f(x w) w^{-1} K(x) w g(x w)^{T} e^{-|x|^{2} / 2} d x & =\int_{\mathbb{R}^{2}} f(x) w^{-1} K\left(x w^{-1}\right) w g(x)^{T} e^{-|x|^{2} / 2} d x \\
& =\int_{\mathbb{R}^{2}} f(x) K(x) g(x)^{T} e^{-|x|^{2} / 2} d x .
\end{aligned}
$$

For the second step change the variable from $x$ to $x w^{-1}$ (note $w^{T}=w^{-1}$ ). Thus we impose the condition

$$
K(x w)=w^{-1} K(x) w .
$$

This implies that it suffices to determine $K$ on the fundamental region $\mathcal{C}_{0}$ and then extend to all of $\Omega$ by using this formula. Set $\partial_{i}:=\frac{\partial}{\partial x_{i}}$. Recall for the scalar situation that the analogous weight function is $h_{\kappa}(x)^{2}$ where $h_{\kappa}(x)=\prod_{v \in R_{+}}|\langle x, v\rangle|^{\kappa(v)}$ and satisfies

$$
\partial_{i} h_{\kappa}(x)=\sum_{v \in R_{+}} \kappa(v) \frac{v_{i}}{\langle x, v\rangle} h_{\kappa}(x), \quad i=1,2 .
$$

Start by solving the equation

$$
\partial_{i} L(x)=\sum_{v \in R_{+}} \kappa(v) \frac{v_{i}}{\langle x, v\rangle} L(x) \sigma_{v}, \quad i=1,2,
$$

for a $2 \times 2$ matrix function $L$ on $\mathcal{C}_{0}$, extended by

$$
L(x w)=L(x) w
$$

(from the facts that $w^{-1} \sigma_{v} w=\sigma_{v w}$ and $\kappa(v w)=\kappa(v)$ it follows that equation (13) is satisfied on all of $\Omega$ ) and set

$$
K(x):=L(x)^{T} L(x),
$$

with the result that $K$ is positive-semidefinite and (note $\sigma_{v}^{T}=\sigma_{v}$ )

$$
\partial_{i} K(x)=\sum_{v \in R_{+}} \kappa(v) \frac{v_{i}}{\langle x, v\rangle}\left(\sigma_{v} K(x)+K(x) \sigma_{v}\right) .
$$

Then for $f, g \in \mathcal{P}_{V}$ (and $i=1,2$ ) we find

$$
\begin{aligned}
-\partial_{i} & \left(f(x) K(x) g(x)^{T} e^{-|x|^{2} / 2}\right) e^{|x|^{2} / 2}=x_{i} f(x) K(x) g(x)^{T}-\left(\partial_{i} f(x)\right) K(x) g(x)^{T} \\
& -f(x)^{T} K(x)\left(\partial_{i} g(x)\right)^{T}-\sum_{v \in R_{+}} \kappa(v) \frac{v_{i}}{\langle x, v\rangle}\left\{f(x) \sigma_{v} K(x) g(x)^{T}+f(x) K(x) \sigma_{v} g(x)^{T}\right\} .
\end{aligned}
$$




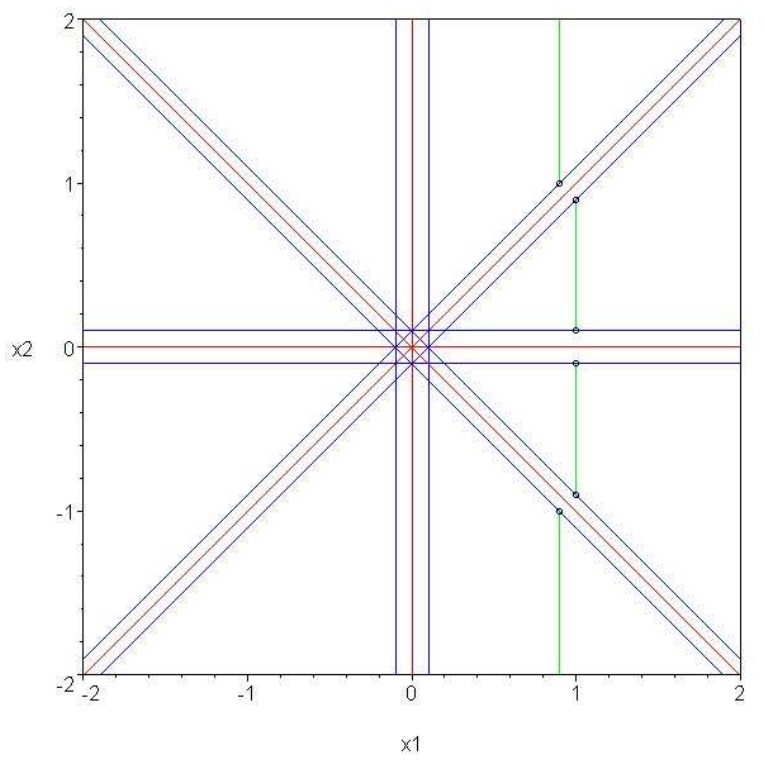

Figure 1. Region of integration.

Consider the second necessary condition (12) $\left\langle\left(x_{i}-\mathcal{D}_{i}\right) f, g\right\rangle_{G}-\left\langle f, \mathcal{D}_{i} g\right\rangle_{G}=0$, that is, the following integral must vanish

$$
\begin{aligned}
\int_{\Omega_{\varepsilon}}\{( & \left.\left.\left(x_{i}-\mathcal{D}_{i}\right) f(x)\right) K(x) g(x)^{T}-f(x) K(x)\left(\mathcal{D}_{i} g(x)\right)^{T}\right\} e^{-|x|^{2} / 2} d x \\
= & -\int_{\Omega_{\varepsilon}} \partial_{i}\left(f(x) K(x) g(x)^{T} e^{-|x|^{2} / 2}\right) d x \\
& +\sum_{v \in R_{+}} \kappa(v) \int_{\Omega_{\varepsilon}} \frac{v_{i}}{\langle x, v\rangle}\left\{f\left(x \sigma_{v}\right) \sigma_{v} K(x) g(x)^{T}+f(x) K(x) \sigma_{v} g\left(x \sigma_{v}\right)^{T}\right\} d x .
\end{aligned}
$$

In the second part, for each $v \in R_{+}$change the variable to $x \sigma_{v}$, then the numerator is invariant, because $\sigma_{v} K\left(x \sigma_{v}\right)=K(x) \sigma_{v}$, and $\left\langle x \sigma_{v}, v\right\rangle=-\langle x, v\rangle$, and thus each term vanishes (note $\Omega_{\varepsilon}$ is $W$-invariant). So establishing the validity of the inner product formula reduces to showing $\lim _{\varepsilon \rightarrow 0_{+}} \int_{\Omega_{\varepsilon}} \partial_{i}\left(f(x) K(x) g(x)^{T} e^{-|x|^{2} / 2}\right) d x=0$ for $i=1,2$. By the polar identity it suffices to prove this for $g=f$. Set $Q(x)=f(x) K(x) f(x)^{T} e^{-|x|^{2} / 2}$.

By symmetry $\left(\sigma_{12}^{+} \mathcal{D}_{1} \sigma_{12}^{+}=\mathcal{D}_{2}\right)$ it suffices to prove the formula for $i=2$. Consider the part of $\Omega_{\varepsilon}$ in $\left\{x_{1}>0\right\}$ as the union of $\left\{x: \varepsilon<\left|x_{2}\right|<x_{1}-\varepsilon\right\}$ and $\left\{x: \varepsilon<x_{1}<\left|x_{2}\right|-\varepsilon\right\}$ (with vertices $(2 \varepsilon, \pm \varepsilon),(\varepsilon, \pm 2 \varepsilon)$ respectively). In the iterated integral evaluate the inner integral over $x_{2}$ on the segments $\left\{\left(x_{1}, x_{2}\right): \varepsilon \leq\left|x_{2}\right| \leq x_{1}-\varepsilon\right\}$ and $\left\{\left(x_{1}-\varepsilon, x_{2}\right): x_{1} \leq\left|x_{2}\right|\right\}$ for a fixed $x_{1}>2 \varepsilon$; obtaining (due to the exponential decay)

$$
\begin{gathered}
-\left(Q\left(x_{1}-\varepsilon, x_{1}\right)-Q\left(x_{1}, x_{1}-\varepsilon\right)\right)-\left(Q\left(x_{1}, \varepsilon\right)-Q\left(x_{1},-\varepsilon\right)\right) \\
-\left(Q\left(x_{1},-x_{1}+\varepsilon\right)-Q\left(x_{1}-\varepsilon,-x_{1}\right)\right) .
\end{gathered}
$$

See Fig. 1 for a diagram of $\Omega_{\varepsilon}$ and a typical inner integral.

By differentiability

$$
\begin{aligned}
& h_{i j}\left(x_{1}, \varepsilon\right)-h_{i j}\left(x_{1},-\varepsilon\right)=C_{1}\left(x_{1}\right) \varepsilon+O\left(\varepsilon^{2}\right), \\
& h_{i j}\left(x_{1}-\varepsilon, x_{1}\right)-h_{i j}\left(x_{1}, x_{1}-\varepsilon\right)=C_{2}\left(x_{1}\right) \varepsilon+O\left(\varepsilon^{2}\right),
\end{aligned}
$$

where $h_{i j}(x)=f_{i}(x) f_{j}(x) e^{-|x|^{2} / 2}$ for $1 \leq i, j \leq 2$; the factors $C_{1}, C_{2}$ depend on $x_{1}$ but there is a global bound $\left|C_{i}\left(x_{1}\right)\right|<C_{0}$ depending only on $f$ because of the exponential decay. Thus 
the behavior of $K\left(x_{1}, \varepsilon\right)$ and $K\left(x_{1}, x_{1}-\varepsilon\right)$ is crucial in analyzing the limit as $\varepsilon \rightarrow 0_{+}$, for $x_{2}=-x_{1}, 0, x_{1}$. It suffices to consider the fundamental region $0<x_{2}<x_{1}$. At the edge $\left\{x_{2}=0\right\}$ corresponding to $\sigma_{2}$

$$
K\left(x_{1},-\varepsilon\right)=K\left(\left(x_{1}, \varepsilon\right) \sigma_{2}\right)=\sigma_{2} K\left(x_{1}, \varepsilon\right) \sigma_{2},
$$

and

$$
\begin{aligned}
& \left(Q\left(x_{1}, \varepsilon\right)-Q\left(x_{1},-\varepsilon\right)\right)=\left(h_{11}\left(x_{1}, \varepsilon\right)-h_{11}\left(x_{1},-\varepsilon\right)\right) K\left(x_{1}, \varepsilon\right)_{11} \\
& \quad+\left(h_{22}\left(x_{1}, \varepsilon\right)-h_{22}\left(x_{1},-\varepsilon\right)\right) K\left(x_{1}, \varepsilon\right)_{22}-2\left(h_{12}\left(x_{1}, \varepsilon\right)+h_{12}\left(x_{1},-\varepsilon\right)\right) K\left(x_{1}, \varepsilon\right)_{12} .
\end{aligned}
$$

At the edge $\left\{x_{1}-x_{2}=0\right\}$ corresponding to $\sigma_{12}^{+}$

$$
K\left(x_{1}-\varepsilon, x_{1}\right)=K\left(\left(x_{1}, x_{1}-\varepsilon\right) \sigma_{12}^{+}\right)=\sigma_{12}^{+} K\left(x_{1}, x_{1}-\varepsilon\right) \sigma_{12}^{+} .
$$

For conciseness set $x^{(0)}=\left(x_{1}, x_{1}-\varepsilon\right)$ and $x^{(1)}=\left(x_{1}-\varepsilon, x_{1}\right)$. Then

$$
\begin{aligned}
& Q\left(x^{(0)}\right)-Q\left(x^{(1)}\right)=2\left(h_{12}\left(x^{(0)}\right)-h_{12}\left(x^{(1)}\right)\right) K\left(x^{(0)}\right)_{12} \\
& +\frac{1}{2}\left(h_{11}\left(x^{(0)}\right)-h_{11}\left(x^{(1)}\right)+h_{22}\left(x^{(0)}\right)-h_{22}\left(x^{(1)}\right)\right)\left(K\left(x^{(0)}\right)_{11}+K\left(x^{(0)}\right)_{22}\right) \\
& +\frac{1}{2}\left(h_{11}\left(x^{(0)}\right)+h_{11}\left(x^{(1)}\right)-h_{22}\left(x^{(0)}\right)-h_{22}\left(x^{(1)}\right)\right)\left(K\left(x^{(0)}\right)_{11}-K\left(x^{(0)}\right)_{22}\right) .
\end{aligned}
$$

To get zero limits as $\varepsilon \rightarrow 0_{+}$some parts rely on the uniform continuity of $h_{i j}$ and bounds on certain entries of $K$, and the other parts require

$$
\lim _{\varepsilon \rightarrow 0_{+}} K\left(x_{1}, \varepsilon\right)_{12}=0, \quad \lim _{\varepsilon \rightarrow 0_{+}}\left(K\left(x_{1}, x_{1}-\varepsilon\right)_{11}-K\left(x_{1}, x_{1}-\varepsilon\right)_{22}\right)=0 .
$$

This imposes various conditions on $K$ near the edges, as described above.

We turn to the solution of the system (13) and rewrite

$$
\begin{aligned}
& \partial_{1} L(x)=L(x)\left\{\frac{k_{1}}{x_{1}}\left[\begin{array}{cc}
-1 & 0 \\
0 & 1
\end{array}\right]+\frac{2 k_{0} x_{2}}{x_{1}^{2}-x_{2}^{2}}\left[\begin{array}{ll}
0 & 1 \\
1 & 0
\end{array}\right]\right\}, \\
& \partial_{2} L(x)=L(x)\left\{\frac{k_{1}}{x_{2}}\left[\begin{array}{cc}
1 & 0 \\
0 & -1
\end{array}\right]-\frac{2 k_{0} x_{1}}{x_{1}^{2}-x_{2}^{2}}\left[\begin{array}{ll}
0 & 1 \\
1 & 0
\end{array}\right]\right\} .
\end{aligned}
$$

The reflections $\sigma_{12}^{-}$and $\sigma_{12}^{+}$were combined into one term: $\frac{(1,-1)}{x_{1}-x_{2}}-\frac{(1,1)}{x_{1}+x_{2}}=\frac{\left(2 x_{2},-2 x_{1}\right)}{x_{1}^{2}-x_{2}^{2}}$. Since $\left(x_{1} \partial_{1}+x_{2} \partial_{2}\right) L(x)=0$ we see that $L$ is positively homogeneous of degree 0 . Because of the homogeneity the system can be transformed to an ordinary differential system by setting $u=\frac{x_{2}}{x_{1}}$. Then the system is transformed to

$$
\frac{d}{d u} L(u)=L(u)\left\{\frac{k_{1}}{u}\left[\begin{array}{cc}
1 & 0 \\
0 & -1
\end{array}\right]-\frac{2 k_{0}}{1-u^{2}}\left[\begin{array}{ll}
0 & 1 \\
1 & 0
\end{array}\right]\right\} .
$$

It follows from this equation that $\frac{d}{d u} \operatorname{det} L(u)=0$. Since the goal is to find a positive-definite matrix $K$ we look for a fundamental solution for $L$, that is $\operatorname{det} L(x) \neq 0$. If $L(x)$ is a solution then so is $M L(x)$ for any nonsingular constant matrix. Thus $K(x)=L(x)^{T} M^{T} M L(x)$ satisfies the differential equation (14) and some other condition must be imposed to obtain the desired (unique) solution for the weight function. The process starts by solving for a row of $L$, say $\left(f_{1}(u), f_{2}(u)\right)$, that is

$$
\frac{d}{d u} f_{1}(u)=\frac{k_{1}}{u} f_{1}(u)-\frac{2 k_{0}}{1-u^{2}} f_{2}(u), \quad \frac{d}{d u} f_{2}(u)=-\frac{2 k_{0}}{1-u^{2}} f_{1}(u)-\frac{k_{1}}{u} f_{2}(u) .
$$


A form of solutions can be obtained by computer algebra, then a desirable solution can be verified. Set

$$
f_{1}(u)=|u|^{k_{1}}\left(1-u^{2}\right)^{-k_{0}} g_{1}\left(u^{2}\right), \quad f_{2}(u)=|u|^{k_{1}}\left(1-u^{2}\right)^{-k_{0}} u g_{2}\left(u^{2}\right),
$$

then the equations become (with $s:=u^{2}$ )

$$
\begin{aligned}
& (s-1) \frac{d}{d s} g_{1}(s)=k_{0} g_{1}(s)+k_{0} g_{2}(s), \\
& s(s-1) \frac{d}{d s} g_{2}(s)=k_{0} g_{1}(s)+\left\{k_{0} s-\left(\frac{1}{2}+k_{1}\right)(s-1)\right\} g_{2}(s),
\end{aligned}
$$

and the solution regular at $s=0$ is

$$
\begin{aligned}
& g_{1}(s)=F\left(-k_{0}, k_{1}+\frac{1}{2}-k_{0} ; k_{1}+\frac{1}{2} ; s\right), \\
& g_{2}(s)=-\frac{k_{0}}{k_{1}+\frac{1}{2}} F\left(1-k_{0}, k_{1}+\frac{1}{2}-k_{0} ; k_{1}+\frac{3}{2} ; s\right) .
\end{aligned}
$$

(We use $F$ to denote the hypergeometric function ${ }_{2} F_{1}$; it is the only type appearing here.) The verification uses two hypergeometric identities (arbitrary parameters $a, b, c$ with $-c \notin \mathbb{N}_{0}$ )

$$
\begin{aligned}
& (s-1) \frac{d}{d s} F(a, b ; c ; s)=-a F(a, b ; c ; s)+\frac{a(c-b)}{c} F(a+1, b ; c+1 ; s), \\
& s(s-1) \frac{d}{d s} F(a+1, b ; c+1 ; s)=(c-b s) F(a+1, b ; c+1 ; s)-c F(a, b ; c ; s) .
\end{aligned}
$$

To get the other solutions we use the symmetry of the system, replace $k_{1}$ by $-k_{1}$ and interchange $f_{1}$ and $f_{2}$. We have a fundamental solution $L(u)$ given by

$$
\begin{aligned}
& L(u)_{11}=|u|^{k_{1}}\left(1-u^{2}\right)^{-k_{0}} F\left(-k_{0}, \frac{1}{2}-k_{0}+k_{1} ; k_{1}+\frac{1}{2} ; u^{2}\right), \\
& L(u)_{12}=-\frac{k_{0}}{k_{1}+\frac{1}{2}}|u|^{k_{1}}\left(1-u^{2}\right)^{-k_{0}} u F\left(1-k_{0}, \frac{1}{2}-k_{0}+k_{1} ; k_{1}+\frac{3}{2} ; u^{2}\right), \\
& L(u)_{21}=-\frac{k_{0}}{\frac{1}{2}-k_{1}}|u|^{-k_{1}}\left(1-u^{2}\right)^{-k_{0}} u F\left(1-k_{0}, \frac{1}{2}-k_{0}-k_{1} ; \frac{3}{2}-k_{1} ; u^{2}\right), \\
& L(u)_{22}=|u|^{-k_{1}}\left(1-u^{2}\right)^{-k_{0}} F\left(-k_{0}, \frac{1}{2}-k_{0}-k_{1} ; \frac{1}{2}-k_{1} ; u^{2}\right) .
\end{aligned}
$$

Observe that $\lim _{u \rightarrow 0_{+}} \operatorname{det} L(u)=1$, thus $\operatorname{det} L(u)=1$ for all $u$. We can write $L$ in the form

$$
L\left(x_{1}, x_{2}\right)=\left[\begin{array}{cc}
\left|x_{2}\right|^{k_{1}} x_{1}^{-k_{1}} & 0 \\
0 & \left|x_{2}\right|^{-k_{1}} x_{1}^{k_{1}}
\end{array}\right]\left[\begin{array}{cc}
c_{11}(x) & \frac{x_{2}}{x_{1}} c_{12}(x) \\
\frac{x_{2}}{x_{1}} c_{21}(x) & c_{22}(x)
\end{array}\right],
$$

where each $c_{i j}$ is even in $x_{2}$ and is real-analytic in $0<\left|x_{2}\right|<x_{1}$. In fact $L(x)$ is thus defined on $\mathcal{C}_{0} \cup \mathcal{C}_{0} \sigma_{2}$. It follows that $K=(M L)^{T} M L$ is integrable near $\left\{x_{2}=0\right\}$ if $\left|k_{1}\right|<\frac{1}{2}$, and $\lim _{\varepsilon \rightarrow 0_{+}} K\left(x_{1}, \varepsilon\right)_{12}=0$ exactly when $M^{T} M$ is diagonal. The standard identity $[8,15.8 .1]$

$$
F(a, b ; c ; u)=(1-u)^{c-a-b} F(c-a, c-b ; c ; u)
$$

shows that there is a hidden symmetry for $k_{0}$

$$
\begin{aligned}
& \left(1-u^{2}\right)^{-k_{0}} F\left(-k_{0}, \frac{1}{2}-k_{0}+k_{1} ; k_{1}+\frac{1}{2} ; u^{2}\right) \\
& =\left(1-u^{2}\right)^{k_{0}} F\left(k_{0}, \frac{1}{2}+k_{0}+k_{1} ; k_{1}+\frac{1}{2} ; u^{2}\right),
\end{aligned}
$$


and similar equations for the other entries of $L$. Consider

$$
\begin{aligned}
& Q\left(x_{1},-\varepsilon\right)-Q\left(x_{1}, \varepsilon\right)=K\left(x_{1}, \varepsilon\right)_{11}\left(h_{11}\left(x_{1},-\varepsilon\right)-h_{11}\left(x_{1}, \varepsilon\right)\right) \\
& \quad-2 K\left(x_{1}, \varepsilon\right)_{12}\left(h_{12}\left(x_{1},-\varepsilon\right)+h_{12}\left(x_{1}, \varepsilon\right)\right)+K\left(x_{1}, \varepsilon\right)_{22}\left(h_{22}\left(x_{1},-\varepsilon\right)-h_{22}\left(x_{1}, \varepsilon\right)\right) .
\end{aligned}
$$

With diagonal $M^{T} M$ we find (note $x_{1}>2 \varepsilon$ in the region, so $\frac{\varepsilon}{x_{1}}<\frac{1}{2}$ )

$$
\begin{aligned}
& K\left(x_{1}, \varepsilon\right)_{11}=O\left(x_{1}^{-2 k_{1}} \varepsilon^{2 k_{1}}\right)+O\left(x_{1}^{2 k_{1}-2} \varepsilon^{2-2 k_{1}}\right), \\
& K\left(x_{1}, \varepsilon\right)_{12}=O\left(x_{1}^{-1-2 k_{1}} \varepsilon^{1+2 k_{1}}\right)+O\left(x_{1}^{-1+2 k_{1}} \varepsilon^{1-2 k_{1}}\right), \\
& K\left(x_{1}, \varepsilon\right)_{22}=O\left(x_{1}^{2 k_{1}} \varepsilon^{-2 k_{1}}\right)+O\left(x_{1}^{-2-2 k_{1}} \varepsilon^{2+2 k_{1}}\right) .
\end{aligned}
$$

By the exponential decay we can assume that the double integral is over the box $\max \left(\left|x_{1}\right|,\left|x_{2}\right|\right) \leq$ $R$ for some $R<\infty$. Note $\int_{2 \varepsilon}^{R}\left(\frac{\varepsilon}{x_{1}}\right)^{\alpha} d x_{1}=\frac{1}{1+\alpha}\left(R^{1-\alpha} \varepsilon^{\alpha}-2^{1-\alpha} \varepsilon\right)$.

These bounds (recall $-\frac{1}{2}<k_{0}, k_{1}<\frac{1}{2}$ ) show that

$$
\lim _{\varepsilon \rightarrow 0_{+}} \int_{2 \varepsilon}^{R}\left(Q\left(x_{1},-\varepsilon\right)-Q\left(x_{1}, \varepsilon\right)\right) d x_{1}=0 .
$$

Next we analyze the behavior of this solution in a neighborhood of $t=1$, that is the ray $\left\{\left(x_{1}, x_{1}\right): x_{1}>0\right\}$. The following identity $[8,15.10 .21]$ is used

$$
\begin{aligned}
F(a+d, a+c ; c+d ; u)= & \frac{\Gamma(c+d) \Gamma(-2 a)}{\Gamma(c-a) \Gamma(d-a)} F(a+d, c+a ; 1+2 a ; 1-u) \\
& +(1-u)^{-2 a} \frac{\Gamma(c+d) \Gamma(2 a)}{\Gamma(c+a) \Gamma(d+a)} F(d-a, c-a ; 1-2 a ; 1-u) .
\end{aligned}
$$

Let

$$
\eta\left(k_{0}, k_{1}\right):=\frac{\Gamma\left(\frac{1}{2}+k_{1}\right) \Gamma\left(2 k_{0}\right)}{\Gamma\left(\frac{1}{2}+k_{0}+k_{1}\right) \Gamma\left(k_{0}\right)}=\frac{2^{2 k_{0}-1}}{\sqrt{\pi}} \frac{\Gamma\left(\frac{1}{2}+k_{1}\right) \Gamma\left(\frac{1}{2}+k_{0}\right)}{\Gamma\left(\frac{1}{2}+k_{0}+k_{1}\right)} ;
$$

the latter equation follows from $\Gamma(2 a) / \Gamma(a)=2^{2 a-1} \Gamma\left(a+\frac{1}{2}\right) / \sqrt{\pi}$ (the duplication formula). We will need the identity

$$
\eta\left(k_{0}, k_{1}\right) \eta\left(-k_{0},-k_{1}\right)+\eta\left(k_{0},-k_{1}\right) \eta\left(-k_{0}, k_{1}\right)=\frac{1}{2},
$$

proved by use of $\Gamma\left(\frac{1}{2}+a\right) \Gamma\left(\frac{1}{2}-a\right)=\frac{\pi}{\cos \pi a}$. Thus

$$
\begin{aligned}
L(u)_{11}= & |u|^{k_{1}}\left\{\eta\left(k_{0}, k_{1}\right)\left(1-u^{2}\right)^{-k_{0}} F\left(-k_{0}, \frac{1}{2}-k_{0}+k_{1} ; 1-2 k_{0} ; 1-u^{2}\right)\right. \\
& \left.+\eta\left(-k_{0}, k_{1}\right)\left(1-u^{2}\right)^{k_{0}} F\left(k_{0}, \frac{1}{2}+k_{0}+k_{1} ; 1+2 k_{0} ; 1-u^{2}\right)\right\},
\end{aligned}
$$

by use of identity (16), and also

$$
\begin{aligned}
L(u)_{12}= & u|u|^{k_{1}}\left\{-\eta\left(k_{0}, k_{1}\right)\left(1-u^{2}\right)^{-k_{0}}{ }_{2} F_{1}\left(1-k_{0}, \frac{1}{2}-k_{0}+k_{1} ; 1-2 k_{0} ; 1-u^{2}\right)\right. \\
& \left.+\eta\left(-k_{0}, k_{1}\right)\left(1-u^{2}\right)^{k_{0}}{ }_{2} F_{1}\left(1+k_{0}, \frac{1}{2}+k_{0}+k_{1} ; 1+2 k_{0} ; 1-u^{2}\right)\right\} .
\end{aligned}
$$

Transform again using (15) to obtain

$$
F\left(1-k_{0}, \frac{1}{2}-k_{0}+k_{1} ; 1-2 k_{0} ; 1-u^{2}\right)=|u|^{-2 k_{1}-1} F\left(-k_{0}, \frac{1}{2}-k_{0}-k_{1} ; 1-2 k_{0} ; 1-u^{2}\right),
$$


and so on. All the hypergeometric functions we use are of one form and it is convenient to introduce

$$
H(a, b ; s):=F\left(a, a+b+\frac{1}{2} ; 2 a+1 ; 1-s\right) .
$$

By using similar transformations as for $L_{11}$ and $L_{12}$ we find

$$
\begin{aligned}
L(u)= & {\left[\begin{array}{cc}
\eta\left(-k_{0}, k_{1}\right) & \eta\left(k_{0}, k_{1}\right) \\
\eta\left(-k_{0},-k_{1}\right) & -\eta\left(k_{0},-k_{1}\right)
\end{array}\right]\left[\begin{array}{cc}
\left(1-u^{2}\right)^{k_{0}} & 0 \\
0 & \left(1-u^{2}\right)^{-k_{0}}
\end{array}\right] } \\
& \times\left[\begin{array}{cc}
H\left(k_{0}, k_{1} ; u^{2}\right) & H\left(k_{0},-k_{1} ; u^{2}\right) \\
H\left(-k_{0}, k_{1} ; u^{2}\right) & -H\left(-k_{0},-k_{1} ; u^{2}\right)
\end{array}\right]\left[\begin{array}{cc}
|u|^{k_{1}} & 0 \\
0 & |u|^{-k_{1}}
\end{array}\right] .
\end{aligned}
$$

Let $\Gamma$ denote the first matrix in the above formula. By direct calculation we find that a necessary condition for

$$
\lim _{\varepsilon \rightarrow 0_{+}}\left(K\left(x_{1}, x_{1}-\varepsilon\right)_{11}-K\left(x_{1}, x_{1}-\varepsilon\right)_{22}\right)=0,
$$

where $K(u)=\left(M^{\prime} \Gamma^{-1} L(u)\right)^{T}\left(M^{\prime} \Gamma^{-1} L(u)\right)$ is that $M^{\prime T} M^{\prime}$ is diagonal. The proof that

$$
\int_{2 \varepsilon}^{R}\left(Q\left(x_{1}, x_{1}-\varepsilon\right)-Q\left(x_{1}-\varepsilon, x_{1}\right)\right) d x_{1} \rightarrow 0
$$

is similar to the previous case; a typical term in $K(u)_{11}-K(u)_{22}$ is

$$
\left(1-u^{2}\right)^{2 k_{0}}\left(H\left(k_{0}, k_{1} ; u^{2}\right)^{2}|u|^{2 k_{1}}-H\left(k_{0},-k_{1} ; u^{2}\right)^{2}|u|^{-2 k_{1}}\right)
$$

which is $O\left((1-u)^{1+2 k_{0}}\right)$, tending to zero as $u \rightarrow 1_{-}$for $\left.\left|k_{0}\right|<\frac{1}{2}\right)$.

It remains to combine the two conditions: $K(u)=(M L(u))^{T}(M L(u))$ and the previous one to find a unique solution for $M: M^{T} M=\left(\Gamma^{-1}\right)^{T} D \Gamma^{-1}$ and both $M^{T} M$ and $D$ are positivedefinite diagonal. Indeed

$$
\begin{aligned}
& D=c\left[\begin{array}{cc}
\eta\left(-k_{0},-k_{1}\right) \eta\left(-k_{0}, k_{1}\right) & 0 \\
0 & \eta\left(k_{0}, k_{1}\right) \eta\left(k_{0},-k_{1}\right)
\end{array}\right], \\
& M^{T} M=2 c\left[\begin{array}{cc}
\eta\left(-k_{0},-k_{1}\right) \eta\left(k_{0},-k_{1}\right) & 0 \\
0 & \eta\left(k_{0}, k_{1}\right) \eta\left(-k_{0}, k_{1}\right)
\end{array}\right],
\end{aligned}
$$

for some $c>0$. Thus the desired matrix weight (in the region $\mathcal{C}_{0}, 0<x_{2}<x_{1}, u=x_{2} / x_{1}$ ) is given by

$$
\begin{aligned}
& K(u)_{11}=d_{1} L(u)_{11}^{2}+d_{2} L(u)_{21}^{2}, \\
& K(u)_{12}=K(u)_{21}=d_{1} L(u)_{11} L(u)_{12}+d_{2} L(u)_{21} L(u)_{22}, \\
& K(u)_{22}=d_{1} L(u)_{12}^{2}+d_{2} L(u)_{22}^{2}
\end{aligned}
$$

where

$$
\begin{aligned}
& d_{1}=c \frac{\Gamma\left(\frac{1}{2}-k_{1}\right)^{2}}{\cos \pi k_{0} \Gamma\left(\frac{1}{2}+k_{0}-k_{1}\right) \Gamma\left(\frac{1}{2}-k_{0}-k_{1}\right)}, \\
& d_{2}=c \frac{\Gamma\left(\frac{1}{2}+k_{1}\right)^{2}}{\cos \pi k_{0} \Gamma\left(\frac{1}{2}+k_{0}+k_{1}\right) \Gamma\left(\frac{1}{2}-k_{0}+k_{1}\right)} .
\end{aligned}
$$




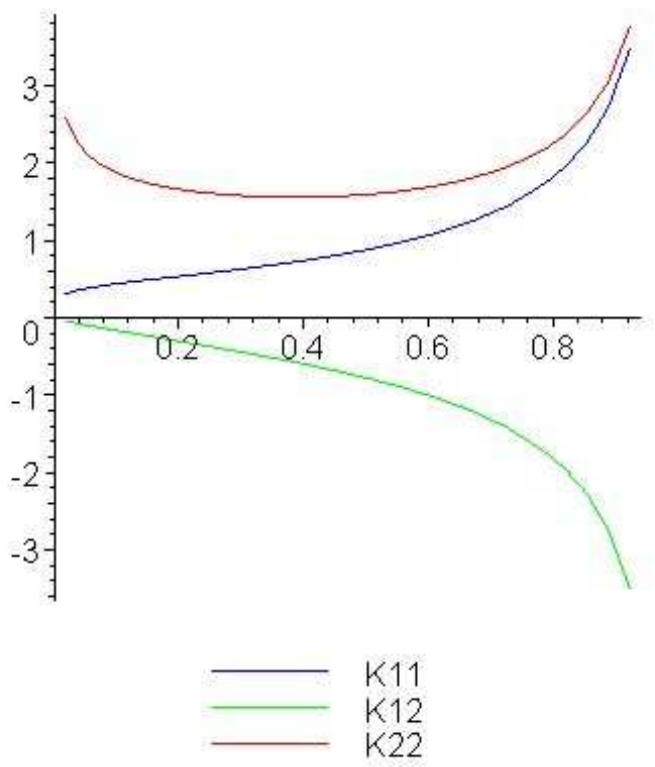

Figure 2. $K, k_{0}=0.3, k_{1}=0.1$.

Also det $K=d_{1} d_{2}=c^{2}\left(1-\tan ^{2} \pi k_{0} \tan ^{2} \pi k_{1}\right)$. The expressions for $K_{i j}$ can be rewritten somewhat by using the transformations (16). Observe that the conditions $-\frac{1}{2}< \pm k_{0} \pm k_{1}<\frac{1}{2}$ are needed for $d_{1}, d_{2}>0$. The normalization constant is to be determined from the condition $\int_{\mathbb{R}^{2}} K(x)_{11} e^{-|x|^{2} / 2} d x=1$. By the homogeneity of $K$ this is equivalent to evaluating $\int_{-\pi}^{\pi} K(\cos \theta, \sin \theta){ }_{11} d \theta$ (or $\int_{0}^{\pi / 4}\left(K_{11}+K_{22}\right)(\cos \theta, \sin \theta) d \theta$ ) (the integral looks difficult because $K_{11}$ involves squares of hypergeometric functions with argument $\left.\tan ^{2} \theta\right)$. Numerical experiments suggest the following conjecture for the normalizing constant

$$
c=\frac{\cos \pi k_{0} \cos \pi k_{1}}{2 \pi} .
$$

We illustrate $K$ for $k_{0}=0.3, k_{1}=0.1$ with plots of $K(\cos \theta, \sin \theta)$ for $0<\theta<\frac{\pi}{4}$. Fig. 2 shows the values of $K_{11}, K_{12}, K_{22}$. For behavior near $x_{1}=x_{2}$ introduce

$$
\sigma=\frac{1}{\sqrt{2}}\left[\begin{array}{cc}
1 & 1 \\
1 & -1
\end{array}\right], \quad \sigma \sigma_{12}^{+} \sigma=\left[\begin{array}{cc}
1 & 0 \\
0 & -1
\end{array}\right]
$$

Fig. 3 displays $\sigma K \sigma$ (thus $(\sigma K \sigma)_{12}=\frac{1}{2}\left(K_{11}-K_{22}\right)$; the $(2,2)$-entry is rescaled by 0.1$)$.

The degenerate cases $k_{0}=0$ and $k_{1}=0$ (when the group aspect reduces to $\boldsymbol{Z}_{2} \times \boldsymbol{Z}_{2}$ ) provide a small check on the calculations: for $k_{0}=0$ the weight is

$$
K(x)=c\left[\begin{array}{cc}
\left|x_{2} / x_{1}\right|^{k_{1}} & 0 \\
0 & \left|x_{1} / x_{2}\right|^{k_{1}}
\end{array}\right]
$$

and for $k_{1}=0$ and by use of the quadratic transformation $F\left(a, a+\frac{1}{2} ; 2 a+1 ; 1-u^{2}\right)=\left(\frac{1+u}{2}\right)^{-2 a}$ (for $u$ near 1$)($ see $[8,15.4 .17])$ we obtain

$$
K(x)=c \sigma\left[\begin{array}{cc}
\left|x_{1}-x_{2}\right|^{k_{0}}\left|x_{1}+x_{2}\right|^{-k_{0}} & 0 \\
0 & \left|x_{1}-x_{2}\right|^{-k_{0}}\left|x_{1}+x_{2}\right|^{k_{0}}
\end{array}\right] \sigma .
$$

An orthogonal basis for $\mathcal{P}_{V}$ for the Gaussian inner product can be given in terms of Laguerre polynomials and the harmonic polynomials from the previous section. Recall from equations (4) 


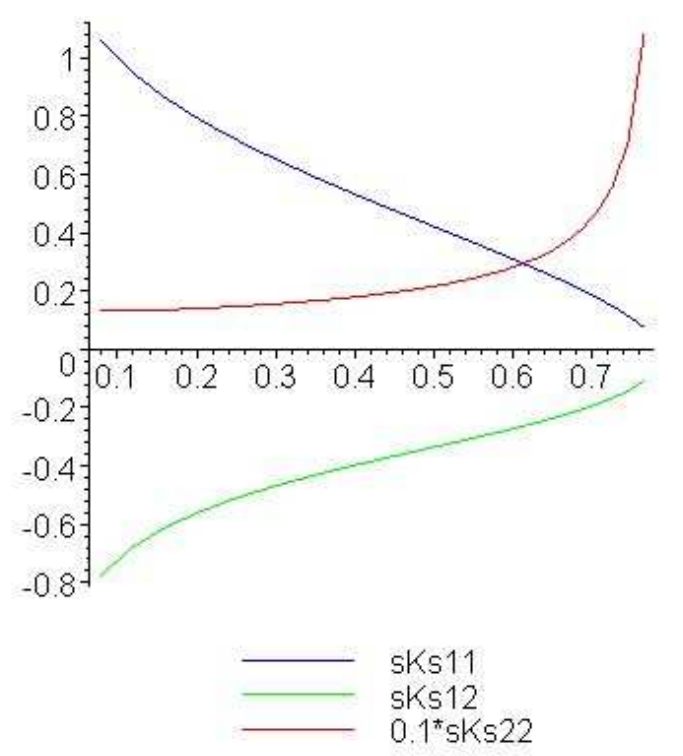

Figure 3. $\sigma K \sigma, k_{0}=0.3, k_{1}=0.1$.

and (6) (specializing $N=2$ and $\gamma(\kappa ; \tau)=0$ ) that

$$
\begin{aligned}
& \Delta_{\kappa}^{k}\left(|x|^{2 m} f(x, t)\right)=4^{k}(-m)_{k}(-m-n)_{k}|x|^{2 m-2 k} f(x, t), \\
& \left\langle|x|^{2 m} f,|x|^{2 m} g\right\rangle_{\tau}=4^{m} m !(n+1)_{m}\langle f, g\rangle_{\tau},
\end{aligned}
$$

for $f, g \in \mathcal{H}_{V, \kappa, n}$. These relations are transferred to the Gaussian inner product

$$
\langle f, g\rangle_{\tau}=\left\langle e^{-\Delta_{\kappa} / 2} f, e^{-\Delta_{\kappa} / 2} g\right\rangle_{G}
$$

by computing

$$
\begin{aligned}
e^{-\Delta_{\kappa} / 2}|x|^{2 m} f(x, t) & =\sum_{j=0}^{m}\left(-\frac{1}{2}\right)^{j} \frac{4^{j}}{j !}(-m)_{j}(-m-n)_{j}|x|^{2 m-2 j} f(x, t) \\
& =(-1)^{m} 2^{m} m ! L_{m}^{(n)}\left(\frac{|x|^{2}}{2}\right) f(x, t),
\end{aligned}
$$

for $f \in \mathcal{H}_{V, \kappa, n}$ and $m \geq 0$; where $L_{m}^{(n)}$ denotes the Laguerre polynomial of degree $m$ and index $n$ (orthogonal for $s^{n} e^{-s} d s$ on $\mathbb{R}_{+}$). Denote $\langle f, f\rangle_{G}$ by $\nu_{G}(f)$; recall $\nu(f)=\langle f, f\rangle_{\tau}$ for $f \in \mathcal{P}_{V}$.

Proposition 8. The polynomials $L_{m}^{(n)}\left(\frac{|x|^{2}}{2}\right) p_{n, i}(x, t)$ for $m, n \geq 0$ and $1 \leq i \leq 4$ (except $i=1,2$ when $n=0)$ are mutually orthogonal in $\langle\cdot, \cdot\rangle_{G}$ and $\nu_{G}\left(L_{m}^{(n)}\left(|x|^{2} / 2\right) p_{n, i}(x, t)\right)=\frac{(n+1)_{m}}{m !} \nu\left(p_{n, i}\right)$.

The factor with $\nu\left(p_{n, i}\right)$ results from a simple calculation. Note $\nu_{G}(f)=\nu(f)$ for any harmonic $f$. Because here $\gamma(\kappa ; \tau)=0$ the harmonic decomposition formula (5) is valid for any parameter values. This is a notable difference from the scalar case $\tau=1$ where $\gamma(\kappa ; 1)=2 k_{0}+2 k_{1}$ and $2 k_{0}+2 k_{1} \neq-1,-2, \ldots$ is required for validity.

Using the same arguments as in the scalar case (see [5, Section 5.7]) we define the Fourier transform (for suitably integrable functions $f$ ). To adapt $E(x, y)$ to vector notation write $E(x, y)=\sum_{i, j=1}^{2} E_{i j}(x, y) s_{i} t_{j}$ and set

$$
\begin{aligned}
& \mathcal{F} f(y)_{l}:=\int_{\mathbb{R}^{2}} \sum_{i, j=1}^{2} E_{l i}(x,-\mathrm{i} y) K(x)_{i j} f_{j}(x) d x_{1} d x_{2}, \quad l=1,2, \\
& \mathcal{F} f(y):=\mathcal{F} f(y)_{1} s_{1}+\mathcal{F} f(y)_{2} s_{2} .
\end{aligned}
$$


For $m, n \geq 0$ and $1 \leq i \leq 4$ let $\phi_{m, n, i}(x)=L_{m}^{(n)}\left(|x|^{2}\right) p_{n, i}(x) e^{-|x|^{2} / 2}$.

Proposition 9. Suppose $m, n \geq 0$ and $1 \leq i \leq 4$ then $\mathcal{F} \phi_{m, n, i}(y)=(-\mathrm{i})^{m+2 n} \phi_{m, n, i}(y)^{*}$. If $f(x)=e^{-|x|^{2} / 2} g(x)$ for $g \in \mathcal{P}_{V}$ then $\mathcal{F}\left(\mathcal{D}_{j} f\right)(y)=i y_{j} \mathcal{F} f(y)$, for $j=1,2$.

This establishes a Plancherel theorem for $\mathcal{F}$ by use of the density (from Hamburger's theorem) of $\operatorname{span}\left\{\phi_{m, n, i}\right\}$ in $L^{2}\left(K(x) d x, \mathbb{R}^{2}\right)$.

\section{Closing remarks}

The well-developed theory of the hypergeometric function allowed us to find the weight function which satisfies both a differential equation and geometric conditions. The analogous problem can be stated for any real reflection group and there are some known results about the differential system (13) (see $[2,3]$ ); it appears some new insights are needed to cope with the geometric conditions. The fact that the Gaussian inner product $\langle\cdot, \cdot\rangle_{G}$ is well-defined supports speculation that Gaussian-type weight functions exist in general settings. However it has not been shown that $K$ can be produced as a product $L^{T} L$, and the effect of the geometry of the mirrors (walls) on the solutions of the differential system is subtle, as seen in the $B_{2}$-case.

\section{Acknowledgements}

This is the expanded version of an invited lecture presented at the Conference on Harmonic Analysis, Convolution Algebras, and Special Functions, TU München, September 10, 2012.

\section{References}

[1] Carter R.W., Finite groups of Lie type. Conjugacy classes and complex characters, Wiley Classics Library, John Wiley \& Sons Ltd., Chichester, 1993.

[2] Dunkl C.F., Differential-difference operators and monodromy representations of Hecke algebras, Pacific J. Math. 159 (1993), 271-298.

[3] Dunkl C.F., Monodromy of hypergeometric functions for dihedral groups, Integral Transform. Spec. Funct. 1 (1993), 75-86.

[4] Dunkl C.F., Opdam E.M., Dunkl operators for complex reflection groups, Proc. London Math. Soc. 86 (2003), 70-108, math.RT/0108185.

[5] Dunkl C.F., Xu Y., Orthogonal polynomials of several variables, Encyclopedia of Mathematics and its Applications, Vol. 81, Cambridge University Press, Cambridge, 2001.

[6] Etingof P., Stoica E., Unitary representations of rational Cherednik algebras, Represent. Theory 13 (2009), 349-370, arXiv:0901.4595.

[7] Griffeth S., Orthogonal functions generalizing Jack polynomials, Trans. Amer. Math. Soc. 362 (2010), 6131-6157, arXiv:0707.0251.

[8] Olver F.W.J., Lozier D.W., Boisvert R.F., Clark C.W. (Editors), NIST handbook of mathematical functions, U.S. Department of Commerce National Institute of Standards and Technology, Washington, DC, 2010. 